\title{
Cliques in graphs with bounded minimum degree
}

\author{
Allan Siu Lun Lo*
}

November 9, 2018

\begin{abstract}
Let $k_{r}(n, \delta)$ be the minimum number of $r$-cliques in graphs with $n$ vertices and minimum degree $\delta$. We evaluate $k_{r}(n, \delta)$ for $\delta \leq 4 n / 5$ and some other cases. Moreover, we give a construction, which we conjecture to give all extremal graphs (subject to certain conditions on $n, \delta$ and $r$ ).
\end{abstract}

\section{Introduction}

Let $f_{r}(n, e)$ be the minimum number of $r$-cliques in graphs of order $n$ and size $e$. Determining $f_{r}(n, e)$ has been a long studied problem. The case $r=3$, that is counting triangles, has been studied by various people. Erdős [3], Lovasz and Simonovits [7] studied the case when $e=\left(\begin{array}{l}n \\ 2\end{array}\right) / 2+l$ with $0<l \leq n / 2$. Fisher [4] considered the situation when $\left(\begin{array}{l}n \\ 2\end{array}\right) / 2 \leq e \leq 2\left(\begin{array}{c}n \\ 2\end{array}\right) / 3$, but it was not until nearly twenty years later that a dramatic breakthrough of Razborov [9] established the asymptotic value of $f_{3}(n, e)$ for a general $e$. The proof of this used the concept of flag algebra developed in [10. Unfortunately, it seemed difficult to generalise Razborov's proof even for $f_{4}(n, e)$. Nikiforov [8] later gave a simple and elegant proof of the asymptotic values of both $f_{3}(n, e)$ and $f_{4}(n, e)$ for general $e$. However, the asymptotic value of $f_{r}(n, e)$ for $r \geq 5$ have not yet been determined, and the best known lower bounds was given Bollobás [2].

In this paper, we are interested in a variant of $f_{r}(n, e)$, where instead of considering the number of edges we consider the minimum degree. Define $k_{r}(n, \delta)$ to be the minimum number of $r$-cliques in

*DPMMS, University of Cambridge, Cambridge CB3 0WB, UK. Email: allan.lo@cantab.net This author is supported by EPSRC. 
graphs of order $n$ with minimum degree $\delta$. In addition, $k_{r}^{r e g}(n, \delta)$ is defined to be the minimum number of $r$-cliques in $\delta$-regular graphs of order $n$. It should be noted that there exist $n$ and $\delta$ such that $k_{r}(n, \delta)=0$, but $k_{r}^{r e g}(n, \delta)>0$. For example, if $r=3, n$ odd and $2 n / 5<\delta n<2$, then it is easy to show that $k_{3}(n, \delta)=0$. However, a theorem of Andrásfai, Erdős and Sós [1] states that every triangle-free graph of order $n$ with minimal degree greater than $2 n / 5$ is bipartite. Since no regular graph with an odd number of vertices can be bipartite, $k_{3}^{r e g}(n, \delta)>0$ for $n$ odd and $2 n / 5<\delta<n / 2$, whilst $k_{3}(n, \delta)=0$. The author [5] evaluated $k_{3}^{r e g}(n, \delta)$ for $n \geq 10^{7}$ odd and $2 n / 5+\sqrt{n} / 5 \leq$ $\delta \leq n / 2$.

Let $\delta=(1-\beta) n$ with $0<\beta \leq 1$ and $p=\left\lceil\beta^{-1}\right\rceil-1$. Throughout this paper, $\beta$ and $\beta n$ are assumed to be a rational and an integer respectively. Note that $p$ is defined so that by Turán's Theorem [11] $k_{r}(n,(1-\beta) n)>0$ for all $n$ (such that $\beta n$ is an integer) if and only if $r \leq p+1$. Since the case $\beta=1$ implies the trivial case $\delta=0$, we may assume that $0<\beta<1$. Furthermore, we consider the cases $1 /(p+1) \leq \beta<1 / p$ separately for positive integers $p$. Hence, the condition $p=2$ is equivalent to $1 / 3 \leq \beta<1 / 2$, that is, $n / 2<\delta \leq$ $2 n / 3$.

Next, we definite a family $\mathcal{G}(n, \beta)$ of graphs, which gives an upper bound on $k_{r}(n, \delta)$, where $\delta=(1-\beta) n$ and integers $r \geq 3$.

Definition 1.1. Let $n$ and $(1-\beta) n$ be positive integers not both odd with $0<\beta<1$. Define $\mathcal{G}(n, \beta)$ to be the family of graphs $G=(V, E)$ of order $n$ satisfying the following properties. There is a partition of $V$ into $V_{0}, V_{1}, \ldots, V_{p-1}$ with $\left|V_{0}\right|=(1-(p-1) \beta) n$ and $\left|V_{i}\right|=\beta n$ for $1 \leq i \leq p-1$, where again $p=\left\lceil\beta^{-1}\right\rceil-1$. For $0 \leq i<j \leq p-1$, the bipartite graph $G\left[V_{i}, V_{j}\right]$ induced by the vertex classes $V_{i}$ and $V_{j}$ is complete. For $1 \leq i \leq p-1$, the subgraph $G\left[V_{i}\right]$ induced by $V_{i}$ is empty and $G\left[V_{0}\right]$ is a $(1-p \beta) n$-regular graph such that the number of triangles in $G\left[V_{0}\right]$ is minimal over all $(1-p \beta) n$-regular graphs of order $\left|V_{0}\right|=(1-(p-1) \beta) n$.

Note that $\mathcal{G}(n, \beta)$ is only defined if $n$ and $(1-\beta) n$ are not both odd. Thus, whenever we mention $\mathcal{G}(n, \beta)$, we automatically assume that $n$ or $(1-\beta) n$ is even. Furthermore, we say $(n, \beta)$ is feasible if $G\left[V_{0}\right]$ is triangle-free for $G \in \mathcal{G}(n, \beta)$. Note that $G\left[V_{0}\right]$ is regular of degree $(1-p \beta) n \leq(1-(p-1) \beta) n / 2=\left|V_{0}\right| / 2$. Thus, if $\left|V_{0}\right|$ is even, then $G\left[V_{0}\right]$ is triangle-free. Therefore, for a given $\beta$, there exist infinitely many choices of $n$ such that $(n, \beta)$ is a feasible pair. If $(n, \beta)$ is not a feasible pair, then $\left|V_{0}\right|$ is odd. Moreover, it is easy to show that $k_{3}\left(G\left[V_{0}\right]\right)=k_{3}^{r e g}\left(n_{0}, \delta_{0}\right)=o\left(n^{3}\right)$, where $n_{0}=\left|V_{0}\right|=(1-(p-1) \beta) n$, $\delta_{0}=(1-p \beta) n$ and $k_{r}(H)$ is the number of $r$-cliques in a graph $H$. 
By Definition 1.1, every $G \in \mathcal{G}(n, \beta)$ is $(1-\beta) n$-regular. In particular, for positive integers $r \geq 3$, the number of $r$-cliques in $G$ is exactly

$$
\begin{aligned}
k_{r}(G) & =g_{r}(\beta) n^{r}+\left(\begin{array}{c}
p-1 \\
r-3
\end{array}\right)(1-p \beta)^{r-3} n^{r-3} k_{3}\left(G\left[V_{0}\right]\right), \\
& =g_{r}(\beta) n^{r}+\left(\begin{array}{c}
p-1 \\
r-3
\end{array}\right)(1-p \beta)^{r-3} n^{r-3} k_{3}^{r e g}\left(n_{0}, \delta_{0}\right),
\end{aligned}
$$

where $n_{0}=(1-(p-1) \beta) n, \delta_{0}=(1-p \beta) n$ and

$$
\begin{aligned}
g_{r}(\beta)= & \left(\begin{array}{c}
p-1 \\
r
\end{array}\right) \beta^{r}+\left(\begin{array}{l}
p-1 \\
r-1
\end{array}\right)(1-(p-1) \beta) \beta^{r-1} \\
& +\frac{1}{2}\left(\begin{array}{l}
p-1 \\
r-2
\end{array}\right)(1-p \beta)(1-(p-1) \beta) \beta^{r-2}
\end{aligned}
$$

with $\left(\begin{array}{l}x \\ y\end{array}\right)$ defined to be 0 if $x<y$ or $y<0$. Since $k_{3}^{r e g}\left(n_{0}, \delta_{0}\right)=o\left(n^{3}\right)$, (11) becomes $k_{r}(G)=\left(g_{r}(\beta)+o(1)\right) n^{r}$. In fact, most of the time, we consider the case when $(n, \beta)$ is feasible, i.e. $k_{3}\left(G\left[V_{0}\right]\right)=0$ and $k_{r}(G)=g_{r}(\beta) n^{r}$. We conjecture that if $(n, \beta)$ is feasible then $\mathcal{G}(n, \beta)$ is the extremal family for $k_{r}(n, \delta)$ with $\delta=(1-\beta) n$ and $3 \leq r \leq$ $p+1=\left\lceil\beta^{-1}\right\rceil$.

Conjecture 1.2. Let $n$ and $\delta$ be positive integers. Then

$$
k_{r}(n, \delta) \geq g_{r}(\beta) n^{r},
$$

where $\delta=(1-\beta) n$ and $r \geq 3$. Moreover, for $3 \leq r \leq p+1=\left\lceil\beta^{-1}\right\rceil$ equality holds if and only if $(n, \beta)$ is feasible and the extremal graphs are members of $\mathcal{G}(n, \beta)$.

By Turán's Theorem [11], the above conjecture is true when $p=1$ or $r>p+1$. If $\beta=1 /(p+1)$ and $(p+1) \mid n$, then $\mathcal{G}(n, 1 /(p+1))$ only consists $T_{p+1}(n)$, the $(p+1)$-partite Turán graph of order $n$. Bollobás 22 proved that if $(p+1) \mid n$ and $e=(1-1 /(p+1)) n^{2} / 2$, then $f_{r}(n, e)=k_{r}\left(T_{p+1}(n)\right)$. Moreover, $T_{p+1}(n)$ is the only graph of order $n$ with $e$ edges and $f_{r}(n, e) r$-cliques. Hence, it is an easy exercise to show that Conjecture 1.2 is true when $\beta=1 /(p+1)$.

It should be noted that since $\mathcal{G}(n, \beta)$ defines a family of regular graphs, we also conjecture that $k_{r}^{r e g}(n, \delta)$ is achieved by $G \in \mathcal{G}(n, \beta)$, where $\delta=(1-\beta) n$. However, we do not address the problem $k_{r}^{r e g}(n, \delta)$ here. For the remainder of the paper, all graphs are also assumed to be of order $n$ with minimum degree $\delta=(1-\beta) n$ unless stated otherwise. 


\section{Main results}

By our previous observation, Conjecture 1.2 is true for the following three cases: $p=1, r>p+1$ and $\delta=(1-1 /(p+1)) n$. That leaves the situation when $3 \leq r \leq p+1$ and $\delta>n / 2$. In Section 3, we prove Conjecture 1.2 for $n / 2<\delta \leq 2 n / 3$, as follows.

Theorem 2.1. Let $n$ and $\delta$ be positive integers with $n / 2<\delta \leq 2 n / 3$. Then

$$
k_{3}(n, \delta) \geq g_{3}(\beta) n^{3},
$$

where $\delta=(1-\beta) n$. Moreover, equality holds if and only if $(n, \beta)$ is feasible and the extremal graphs are members of $\mathcal{G}(n, \beta)$.

The ideas in the proof, which is short, form the framework for our other results. The next simplest case is that of $K_{p+2}$-free graphs. Notice that, by the definition of $p, G$ must contain $K_{p+1}$ 's but need not contain $K_{p+2}$. Conjecture 1.2 is proved for $K_{p+2}$-free graphs by the next theorem.

Theorem 2.2. Let $n$ and $\delta$ be positive integers. Let $G$ be a $K_{p+2}$-free graph of order $n$ with minimum degree $\delta$, where $\delta=(1-\beta) n$ and $p=\left\lceil\beta^{-1}\right\rceil-1$. Then,

$$
k_{r}(G) \geq g_{r}(\beta) n^{r}
$$

for positive integers $r$. Moreover, for $3 \leq r \leq p+1$ equality holds if and only if $(n, \beta)$ is feasible, and the extremal graphs are members of $\mathcal{G}(n, \beta)$.

Theorem 2.2 is proved in Section 5, after some notations and basic inequalities have been set up in Section 4, It shows that the difficult in proving Conjecture 1.2 is in handling $(p+2)$-cliques. We discuss this situation in Section 6 for the case $p=3$, and by a detailed analysis of 5 -cliques in Section 7, proving Conjecture 1.2 for $2 n / 3<\delta \leq 3 n / 4$, as follows.

Theorem 2.3. Let $n$ and $\delta$ be positive integers with $2 n / 3<\delta \leq 3 n / 4$. Then

$$
k_{r}(n, \delta) \geq g_{r}(\beta) n^{r},
$$

for positive integers $r$ and $\delta=(1-\beta) n$. Moreover, for $3 \leq r \leq 4$ equality holds if and only if $(n, \beta)$ is feasible and the extremal graphs are members of $\mathcal{G}(n, \beta)$.

This theorem is the hardest in the paper. We have in fact proved Conjecture 1.2 for $3 n / 4<\delta \leq 4 n / 5$ by a similar argument. It is too complicated to be included in this paper, but it can be found 
in [6]. For each positive integer $p \geq 5$, it is likely that by following the arguments in the proof of Theorem 2.3 one could construct a proof for Conjecture 1.2 when $(1-1 / p) n<\delta \leq(1-1 /(p+1)) n$.

We give two more results in support of Conjecture 1.2 in Section 8 and Section 9, The first is that for every positive integer $p$, Conjecture 1.2 holds for a positive proportion of values of $\delta$.

Theorem 2.4. For every positive integer $p$, there exists a (calculable) constant $\epsilon_{p}>0$ so that if $n$ and $\delta$ are positive integers such that $\left(1-1 /(p+1)-\epsilon_{p}\right) n<\delta \leq(1-1 /(p+1)) n$, then

$$
k_{r}(n, \delta) \geq g_{r}(\beta) n^{r},
$$

for positive integers $r$ and $\delta=(1-\beta) n$. Moreover, for $3 \leq r \leq p+1$ equality holds if and only if $(n, \beta)$ is feasible and the extremal graphs are members of $\mathcal{G}(n, \beta)$.

Finally, using a different argument, we can show that Conjecture 1.2 holds in the case $r=p+1$ (the largest value of $r$ for which $r$-cliques are guaranteed).

Theorem 2.5. Let $n$ and $\delta$ be positive integers. Then

$$
k_{p+1}(n, \delta) \geq g_{p+1}(\beta) n^{p+1},
$$

where $\delta=(1-\beta) n$ and $p=\left\lceil\beta^{-1}\right\rceil-1$. Moreover, equality holds if and only if $(n, \beta)$ is feasible and the extremal graphs are members of $\mathcal{G}(n, \beta)$.

\section{Proof of Theorem 2.1}

Here we prove Theorem 2.1, that is Conjecture 1.2 for $n / 2<\delta \leq 2 n / 3$, so $1 / 3 \leq \beta<1 / 2$ and $p=2$.

Proof of Theorem 2.1. Let $G$ be a graph of order $n$ with minimum degree $\delta$. Since $G$ has at least $\delta n / 2=(1-\beta) n^{2} / 2$ edges,

$$
(1-2 \beta) \beta n k_{2}(G) \geq(1-2 \beta)(1-\beta) \beta n^{3} / 2=g_{3}(\beta) n^{3} .
$$

Thus, in proving the inequality in Theorem 2.1, it is enough to show that $k_{3}(G) \geq(1-2 \beta) \beta n k_{2}(G)$.

For an edge $e$, define $d(e)$ to be the number of triangles containing $e$ and write $D(e)=d(e) / n$. Clearly,

$$
n \sum_{e \in E(G)} D(e)=\sum d(e)=3 k_{3}(G) .
$$


In addition, $D(e) \geq 1-2 \beta$ for each edge $e$, because each vertex in $G$ misses at most $\beta n$ vertices. Since $\beta<1 / 2, D(e)>0$ for all $e \in E(G)$ and so every edge is contained in a triangle. Let $T$ be a triangle in $G$. Similarly, define $d(T)$ to be the number of 4-cliques containing $T$ and write $D(T)=d(T) / n$. We claim that

$$
\sum_{e \in E(T)} D(e) \geq 2-3 \beta+D(T) .
$$

Let $n_{i}$ be the number vertices in $G$ with exactly $i$ neighbours in $T$ for $i=0,1,2,3$. Clearly, $n=n_{0}+n_{1}+n_{2}+n_{3}$. By counting the number of edges incident with $T$, we obtain

$$
3(1-\beta) n \leq \sum_{v \in V(T)} d(v)=3 n_{3}+2 n_{2}+n_{1} \leq 2 n_{3}+n_{2}+n .
$$

On the other hand, $n_{3}=d(T)$ and $n_{2}+3 n_{3}=\sum_{e \in E(G)} d(e)$. Hence, (22) holds. Notice that if equality holds in (2) then $d(v)=(1-\beta) n$ for all $v \in T$.

For an edge $e$, define $D_{-}(e)=\min \{D(e), \beta\}$. We claim that

$$
\sum_{e \in E(T)} D_{-}(e) \geq 2-3 \beta
$$

for every triangle $T$. If $D(e)=D_{-}(e)$ for each edge $e$ in $T$, then (4) holds by (2). Otherwise, there exists $e_{0} \in E(T)$ such that $D\left(e_{0}\right) \neq$ $D_{-}\left(e_{0}\right)$. This means that $D_{-}\left(e_{0}\right)=\beta$. Recall that for the other two edges $e$ in $T, D(e) \geq 1-2 \beta$, so $\sum D_{-}(e) \geq \beta+2(1-2 \beta)=2-3 \beta$. Hence, (4) holds for every triangle $T$.

Next, by summing (4) over all triangles $T$ in $G$, we obtain

$$
n \sum_{e \in E(G)} D_{-}(e) D(e)=\sum_{T} \sum_{e \in E(T)} D_{-}(e) \geq(2-3 \beta) k_{3}(G) .
$$

We are going to bound $\sum D_{-}(e) D(e)$ above in terms of $\sum D(e)$, which is equal to $3 k_{3}(G) / n$, by the following proposition.

Proposition 3.1. Let $\mathcal{A}$ be a finite set. Suppose $f, g: \mathcal{A} \rightarrow \mathbb{R}$ with $f(a) \leq M$ and $g(a) \geq m$ for all $a \in \mathcal{A}$. Then

$$
\sum_{a \in \mathcal{A}} f(a) g(a) \leq m \sum_{a \in \mathcal{A}} f(a)+M \sum_{a \in \mathcal{A}} g(a)-m M|\mathcal{A}|,
$$

with equality if and only if for each $a \in \mathcal{A}, f(a)=M$ or $g(a)=m$.

Proof. Observe that $\sum_{a \in \mathcal{A}}(M-f(a))(g(a)-m) \geq 0$. 
Recall that $D(e) \geq 1-2 \beta$ and $D_{-}(e) \leq \beta$. By Proposition 3.1 taking $\mathcal{A}=E(G), f=D_{-}, g=D, M=\beta$ and $m=1-2 \beta$, we have

$$
\begin{aligned}
n \sum_{e \in E(G)} D(e) D_{-}(e) & \leq(1-2 \beta) n \sum_{e \in E(G)} D_{-}(e)+\beta n \sum_{e \in E(G)} D(e)-(1-2 \beta) \beta n k_{2}(G) \\
& \leq(1-\beta) n \sum_{e \in E(G)} D(e)-(1-2 \beta) \beta n k_{2}(G) \\
n \sum_{e \in E(G)} D(e) D_{-}(e) & \leq 3(1-\beta) k_{3}(G)-(1-2 \beta) \beta n k_{2}(G) .
\end{aligned}
$$

After substitution of (77) into (5) and rearrangement, we have

$$
k_{3}(G) \geq(1-2 \beta) \beta k_{2}(G) n .
$$

Thus, we have proved the inequality in Theorem 2.1 .

Now suppose equality holds, i.e. $k_{3}(G)=(1-2 \beta) \beta k_{2}(G) n$. This means that equality holds in (6), so (since $\beta<1 / 2) D(e)=D_{-}(e)$ for all $e \in E(G)$. Because equality holds in (4), $\sum_{e \in E(T)} D(e)=2-3 \beta$ for triangles $T$. Hence, $D(T)=0$ for every triangle $T$ by (2), so $G$ is $K_{4^{-}}$ free. In addition, by the remark following (2), $G$ is $(1-\beta) n$-regular, because every vertex lies in a triangle as $D(e)>0$ for all edges $e$. Since equality holds in Proposition 3.1, either $D(e)=1-2 \beta$ or $D(e)=\beta$ for each edge $e$. Recall that equality holds for (2), so every triangle $T$ contains exactly one edge $e_{1}$ with $D\left(e_{1}\right)=\beta$ and two edges, $e_{2}$ and $e_{3}$, with $D\left(e_{2}\right)=D\left(e_{3}\right)=1-\beta$. Pick an edge $e$ with $D(e)=\beta$ and let $W$ be the set of common neighbours of the end vertices of $e$, so $|W|=\beta n$. Clearly $W$ is an independent set, otherwise $G$ contains a $K_{4}$. For each $w \in W, d(w)=(1-\beta) n$ implies $N(w)=V(G) \backslash W$. Therefore, $G[V(G) \backslash W]$ is $(1-2 \beta) n$-regular. If there is a triangle $T$ in $G[V(G) \backslash W]$, then $T \cup w$ forms a $K_{4}$ for $w \in W$. This contradicts the assumption that $G$ is $K_{4}$-free, so $G[V(G) \backslash W]$ is triangle-free. Hence, $G$ is a member of $\mathcal{G}(n, \beta)$ and $(n, \beta)$ is feasible. Therefore, the proof is complete.

\section{Degree of a clique}

Denote the set of $t$-cliques in $G[U]$ by $\mathcal{K}_{t}(U)$ and write $k_{r}(U)$ for $\left|\mathcal{K}_{r}(U)\right|$. If $U=V(G)$, we simply write $\mathcal{K}_{r}$ and $k_{r}$.

Define the degree $d(T)$ of a $t$-clique $T$ to be the number of $(t+1)$ cliques containing $T$. In other words, $d(T)=\left|\left\{S \in \mathcal{K}_{t+1}: T \subset S\right\}\right|$. If $t=1$, then $d(v)$ coincides with the ordinary definition of the degree for a vertex $v$. If $t=2$, then $d(u v)$ is the number of common neighbours 
of the end vertices of the edge $u v$, that is the codegree of $u$ and $v$. Clearly, $\sum_{T \in \mathcal{K}_{t}} d(T)=(t+1) k_{t+1}$ for $t \geq 1$. For convenience, we write $D(T)$ to denote $d(T) / n$.

Recall that $p=\left\lceil\beta^{-1}\right\rceil-1$ and $1 /(p+1) \leq \beta<1 / p$. Let $G_{0} \in$ $\mathcal{G}(n, \beta)$ with $(n, \beta)$ feasible. Let $T$ be a $t$-clique in $G_{0}$. It is natural to see that there are three types of cliques according to $\left|T \cap V_{0}\right|$. However, if we consider $d(T)$, then there are only two types. To be precise

$$
D(T)= \begin{cases}1-t \beta & \text { if }\left|V(T) \cap V_{0}\right|=0,1 \text { and } \\ (p-t+1) \beta & \text { if }\left|V(T) \cap V_{0}\right|=2,\end{cases}
$$

for $T \in \mathcal{K}_{t}\left(G_{0}\right)$ and $2 \leq t \leq p+1$. Next, define the functions $D_{+}$and $D_{-}$as follows. For a graph $G$ with minimum degree $\delta=(1-\beta) n$, define

$$
\begin{aligned}
& D_{-}(T)=\min \{D(T),(p-t+1) \beta\}, \text { and } \\
& D_{+}(T)=D(T)-D_{-}(T)=\max \{0, D(T)-(p-t+1) \beta\}
\end{aligned}
$$

for $T \in \mathcal{K}_{t}$ and $1 \leq t \leq p+1$. We say that a clique $T$ is heavy if $D_{+}(T)>0$. The graph $G$ is said to be heavy-free if and only if $G$ does not contain any heavy cliques. Now, we study some basic properties of $D(T), D_{-}(T)$ and $D_{+}(T)$.

Lemma 4.1. Let $0<\beta<1$ and $p=\left\lceil\beta^{-1}\right\rceil-1$. Suppose $G$ is a graph of order $n$ with minimum degree $(1-\beta) n$. Suppose $S \in \mathcal{K}_{s}$ and $T \in \mathcal{K}_{t}(S)$ for $1 \leq t<s$. Then

(i) $D(S) \geq 1-s \beta$,

(ii) $D(S) \geq D(T)-(s-t) \beta$,

(iii) for $s \leq p+1, D_{+}(T) \leq D_{+}(S) \leq D_{+}(T)+(s-t) \beta$,

(iv) if $T$ is heavy and $s \leq p+1$ then $S$ is heavy, and

(v) if $T$ is not heavy and $s \leq p+1$, then $D_{+}(S) \leq(s-t) \beta$. In particular, if $t=s-1 \leq p$, then $D_{+}(S) \leq \beta$.

Moreover, $G$ is $K_{p+2}$-free if and only if $G$ is heavy-free.

Proof. For each $v \in S$, there are at most $\beta n$ vertices not joined to $v$. Hence, $D(S) \geq 1-s \beta$, so $(i)$ is true. Similarly, consider the vertices in $S \backslash T$, so (ii) is also true. If $s \leq p+1$ and $D_{+}(T)>0$, then we have

$$
\begin{aligned}
D_{+}(S)+(p-s+1) \beta & \geq D(S) \\
& \geq D(T)-(s-t) \beta \\
& =D_{+}(T)+(p-t+1) \beta-(s-t) \beta,
\end{aligned}
$$


so the left inequality of $(i i i)$ is true. Since $D(S) \leq D(T)$, the right inequality of (iii) is also true by the definition of $D_{+}(S)$ and $D_{+}(T)$. Hence, $(i v)$ and $(v)$ are true by the left and right inequality in (iii) respectively. Notice that $D(U)=D_{+}(U)$ for $U \in \mathcal{K}_{p+1}$. Hence, by (iv), $G$ is $K_{p+2}$-free if and only if $G$ is heavy-free.

Now we prove the generalised version of (2), that is, the sum of degrees of $t$-subcliques in a $s$-clique.

Lemma 4.2. Let $0<\beta<1$. Let $s$ and $t$ be integers with $2 \leq t<s$. Suppose $G$ is a graph of order $n$ with minimum degree $(1-\beta) n$. Then

$$
\sum_{T \in \mathcal{K}_{t}(S)} D(T) \geq(1-\beta) s\left(\begin{array}{c}
s-2 \\
t-1
\end{array}\right)-(t-1)\left(\begin{array}{c}
s-1 \\
t
\end{array}\right)+\left(\begin{array}{c}
s-2 \\
t-2
\end{array}\right) D(S)
$$

for $S \in \mathcal{K}_{s}$. Moreover, if equality holds, then $d(v)=(1-\beta) n$ for all $v \in S$.

Proof. Let $n_{i}$ be the number of vertices with exactly $i$ neighbours in $S$. The following three equations :

$$
\begin{aligned}
\sum_{i} n_{i} & =n \\
\sum_{i} i n_{i} & =\sum_{v \in V(S)} d(v) \geq s(1-\beta) n, \\
\sum_{i}\left(\begin{array}{l}
i \\
t
\end{array}\right) n_{i} & =\sum_{T \in \mathcal{K}_{t}(S)} D(T) n,
\end{aligned}
$$

follow from a count of the number of vertices, edges and $(t+1)$-cliques respectively. Next, by considering $(t-1)\left(\begin{array}{c}s-1 \\ t\end{array}\right)(\sqrt[8]{8})-\left(\begin{array}{c}s-2 \\ t-1\end{array}\right)(96)+$ (10), we have

$\sum_{T \in \mathcal{K}_{t}(S)} D(T) n \geq\left((1-\beta) s\left(\begin{array}{c}s-2 \\ t-1\end{array}\right)-(t-1)\left(\begin{array}{c}s-1 \\ t\end{array}\right)\right) n+\sum_{0 \leq i \leq s} x_{i} n_{i}$,

where $x_{i}=\left(\begin{array}{l}i \\ t\end{array}\right)+(t-1)\left(\begin{array}{c}s-1 \\ t\end{array}\right)-i\left(\begin{array}{c}s-2 \\ t-1\end{array}\right)$. Notice that $x_{i}=x_{i+1}+\left(\begin{array}{c}s-2 \\ t-1\end{array}\right)-$ $\left(\begin{array}{c}i \\ t-1\end{array}\right) \geq x_{i+1}$ for $0 \leq i \leq s-2$. For $i=s-1$, we have

$$
\begin{aligned}
x_{s-1} & =\left(\begin{array}{c}
s-1 \\
t
\end{array}\right)+(t-1)\left(\begin{array}{c}
s-1 \\
t
\end{array}\right)-(s-1)\left(\begin{array}{c}
s-2 \\
t-1
\end{array}\right) \\
& =t\left(\begin{array}{c}
s-1 \\
t
\end{array}\right)-(s-1)\left(\begin{array}{c}
s-2 \\
t-1
\end{array}\right)=0 .
\end{aligned}
$$


For $i=s, n_{s}=D(S) n$ and

$$
\begin{aligned}
x_{s} & =\left(\begin{array}{l}
s \\
t
\end{array}\right)+(t-1)\left(\begin{array}{c}
s-1 \\
t
\end{array}\right)-s\left(\begin{array}{l}
s-2 \\
t-1
\end{array}\right) \\
& =t\left(\begin{array}{c}
s-1 \\
t
\end{array}\right)+\left(\begin{array}{c}
s-1 \\
t-1
\end{array}\right)-s\left(\begin{array}{c}
s-2 \\
t-1
\end{array}\right) \\
& =(s-t+1)\left(\begin{array}{l}
s-1 \\
t-1
\end{array}\right)-s\left(\begin{array}{c}
s-2 \\
t-1
\end{array}\right) \\
& =(s-t+1)\left(\begin{array}{l}
s-2 \\
t-2
\end{array}\right)-(t-1)\left(\begin{array}{l}
s-2 \\
t-1
\end{array}\right) \\
& =\left(\begin{array}{c}
s-2 \\
t-2
\end{array}\right) .
\end{aligned}
$$

In particular, if equality holds in the lemma, then equality holds in (9). This means that $d(v)=(1-\beta) n$ for all $v \in S$.

Most of the time, we are only interested in the case when $s=t+1$. Hence, we state the following corollary.

Corollary 4.3. Let $0<\beta<1$. Suppose $G$ is a graph or order $n$ with minimum degree $(1-\beta) n$. Then

$$
\sum_{T \in \mathcal{K}_{t}(S)} D(T) \geq 2-(t+1) \beta+(t-1) D(S)
$$

for $S \in \mathcal{K}_{t+1}$ and integer $t \geq 2$. Moreover, if equality holds, then $d(v)=(1-\beta) n$ for all $v \in S$.

In the next lemma, we show that the functions $D$ in Lemma 4.2 can be replaced with $D_{-}$.

Lemma 4.4. Let $0<\beta<1$ and $p=\left\lceil\beta^{-1}\right\rceil-1$. Let $s$ and $t$ be integers with $2 \leq t<s \leq p+1$. Suppose $G$ is a graph of order $n$ with minimum degree $(1-\beta) n$. Then, for $S \in \mathcal{K}_{s}$

$\sum_{T \in \mathcal{K}_{t}(S)} D_{-}(T) \geq(1-\beta) s\left(\begin{array}{c}s-2 \\ t-1\end{array}\right)-(t-1)\left(\begin{array}{c}s-1 \\ t\end{array}\right)+\left(\begin{array}{c}s-2 \\ t-2\end{array}\right) D_{-}(S)$.

Proof. Since $D_{+}(S) \geq D_{+}(T)$ for every $T \in \mathcal{K}_{t}(S)$ by Lemma4.1 (iii), there is nothing to prove by Lemma 4.2 if there are at most $\left(\begin{array}{c}s-2 \\ t-2\end{array}\right)$ heavy $t$-cliques in $S$. Now suppose there are more than $\left(\begin{array}{c}s-2 \\ t-2\end{array}\right)$ heavy $t$ cliques in $S$. In particular, $S$ contains a heavy $t$-clique, so $S$ is itself heavy with $D_{-}(S)=(p+1-s) \beta$ by Lemma 4.1 (iv). Thus, the right hand side of the inequality is $\left(\begin{array}{c}s \\ t\end{array}\right)(1-t \beta)+\left(\begin{array}{c}s-2 \\ t-2\end{array}\right)((p+1) \beta-1)$. By Lemma $4.1(i)$ we have that $D_{-}(T) \geq(1-t \beta)$ for $T \in \mathcal{K}_{t}(S)$. 
Furthermore, by Lemma 4.1 (iv) $D_{-}(T)=(p-t+1) \beta$ if $T$ is heavy, so summing $D_{-}(T)$ over $T \in \mathcal{K}_{t}(S)$ gives

$$
\begin{aligned}
\sum_{T \in \mathcal{K}_{t}(S)} D_{-}(T) & \geq k_{t}^{+}(S)(p-t+1) \beta+\left(\left(\begin{array}{l}
s \\
t
\end{array}\right)-k_{t}^{+}(S)\right)(1-t \beta) \\
& =\left(\begin{array}{l}
s \\
t
\end{array}\right)(1-t \beta)+k_{t}^{+}(S)((p+1) \beta-1) .
\end{aligned}
$$

This completes the proof of the lemma.

Define the function $\widetilde{D}: \mathcal{K}_{t+1} \rightarrow \mathbb{R}$ such that

$$
\widetilde{D}(S)=\sum_{T \in \mathcal{K}_{t}(S)} D_{-}(T)-\left(2-(t+1) \beta+(t-1) D_{-}(S)\right)
$$

for $S \in \mathcal{K}_{t+1}$ and $2 \leq t \leq p$. Hence, for $s=t+1$, Lemma 4.4 gives the following corollary.

Corollary 4.5. Let $0<\beta<1$ and $p=\left\lceil\beta^{-1}\right\rceil-1$. Let $t$ be integer with $2 \leq t \leq p$. Suppose $G$ is a graph of order $n$ with minimum degree $(1-\beta) n$. Then $\widetilde{D}(S) \geq 0$ for $S \in \mathcal{K}_{t+1}$.

Next, we bound $\sum_{S \in \mathcal{K}_{t+1}} \widetilde{D}(S)$ from above using Proposition 3.1

Lemma 4.6. Let $0<\beta<1$ and $p=\left\lceil\beta^{-1}\right\rceil-1$. Let $t$ be an integer with $2 \leq t \leq p$. Suppose $G$ is a graph of order $n$ with minimum degree $(1-\beta) n$. Then

$$
\begin{aligned}
& \sum_{S \in \mathcal{K}_{t+1}} \widetilde{D}(S) \leq(t-1+(p-2 t+2)(t+1) \beta) k_{t+1}+(t-1) \sum_{S \in \mathcal{K}_{t+1}} D_{+}(S) \\
& -(1-t \beta)(p-t+1) \beta n k_{t}-(t-1)(t+2) \frac{k_{t+2}}{n}-(1-t \beta) n \sum_{T \in \mathcal{K}_{t}} D_{+}(T) .
\end{aligned}
$$

Moreover, equality holds if and only if for each $T \in \mathcal{K}_{t}$, either $D_{-}(T)=$ $1-t \beta$ or $D_{-}(T)=(p-t+1) \beta$.

Proof. Notice that the sum $\widetilde{D}(S)$ over $S \in \mathcal{K}_{t+1}$ is equal to

$$
\sum_{S \in \mathcal{K}_{t+1}} \sum_{T \in \mathcal{K}_{t}(S)} D_{-}(T)-(2-(t+1) \beta) k_{t+1}-(t-1) \sum_{S \in \mathcal{K}_{t+1}} D_{-}(S) .
$$

Consider each each term separately. Since $D(S)=D_{-}(S)+D_{+}(S)$,

$$
\sum_{S \in \mathcal{K}_{t+1}} D_{-}(S)=\sum_{S \in \mathcal{K}_{t+1}} D(S)-\sum_{S \in \mathcal{K}_{t+1}} D_{+}(S)=\frac{(t+2) k_{t+2}}{n}-\sum_{S \in \mathcal{K}_{t+1}} D_{+}(S) .
$$


By interchanging the order of summations, we have

$$
\sum_{S \in \mathcal{K}_{t+1}} \sum_{T \in \mathcal{K}_{t}(S)} D_{-}(T)=n \sum_{T \in \mathcal{K}_{t}} D_{-}(T) D(T),
$$

and by Proposition 3.1 taking $\mathcal{A}=\mathcal{K}_{t}, f=D_{-}, g=D, M=(p-t+$ 1) $\beta$ and $m=1-t \beta$

$$
\begin{aligned}
& \leq(1-t \beta) n \sum_{T \in \mathcal{K}_{t}} D_{-}(T)+(p-t+1) \beta n \sum_{T \in \mathcal{K}_{t}} D(T)-(1-t \beta)(p-t+1) \beta n k_{t} \\
& =(1+(p-2 t+1) \beta) n \sum D(T)-(1-t \beta) n \sum D_{+}(T)-(1-t \beta)(p-t+1) \beta n k_{t} \\
& =(1+(p-2 t+1) \beta)(t+1) k_{t+1}-(1-t \beta) n \sum D_{+}(T)-(1-t \beta)(p-t+1) \beta n k_{t} .
\end{aligned}
$$

Hence, substituting these identities back into (11), we obtain the desired inequality in the lemma.

By Proposition 3.1, equality holds if and only if for each $T \in \mathcal{K}_{t}$, either $D(T)=1-t \beta$ or $D_{-}(T)=(p-t+1) \beta$.

To keep our calculations simple, we are going to establish a few relationships between $g_{t}(\beta)$ and $g_{t+1}(\beta)$ in the next lemma.

Lemma 4.7. Let $0<\beta<1$ and $p=\left\lceil\beta^{-1}\right\rceil-1$. Let $t$ be an integer with $2 \leq t \leq p$. Then

$$
\begin{aligned}
(t+1) g_{t+1}(\beta)= & (1-t \beta) g_{t}(\beta) \\
& +\frac{1}{2}\left(\begin{array}{c}
p-1 \\
t-2
\end{array}\right)((p+1) \beta-1)(1-(p-1) \beta)(1-p \beta) \beta^{t-2} \\
g_{t+1}(\beta)= & \frac{(1-t \beta)(p-t+1) \beta g_{t}(\beta)+(t-1)(t+2) g_{t+2}(\beta)}{t-1+(t+1)(p-2 t+2) \beta}
\end{aligned}
$$

Moreover

$$
\frac{g_{p}(\beta)}{g_{p+1}(\beta)}=\frac{1}{\beta}\left(1+\frac{\beta g_{p-1}\left(\beta^{\prime}\right)}{(1-\beta) g_{p}\left(\beta^{\prime}\right)}\right),
$$

where $\beta^{\prime}=\beta /(1-\beta)$.

Proof. We fix $\beta$ (and $p$ ) and write $g_{t}$ to denote $g_{t}(\beta)$. Pick $n$ such that $(n, \beta)$ is feasible and let $G \in \mathcal{G}(n, \beta)$ with partition classes $V_{0}, V_{1}, \ldots, V_{p-1}$ as described in Definition 1.1. Thus, for $T \in \mathcal{K}_{t}, D(T)=1-t \beta$ or $D(T)=(p-t+1) \beta$. Since $D(T)=(p-t+1) \beta$ if and only if $\left|V(T) \cap V_{0}\right|=2$, there are exactly

$$
\frac{1}{2}\left(\begin{array}{c}
p-1 \\
t-2
\end{array}\right)(1-(p-1) \beta)(1-p \beta) \beta^{t-2} n^{t}
$$


$t$-cliques $T$ with $D(T)=(p-t+1) \beta$. Also, we have

$$
(t+1) g_{t+1} n^{t+1}=(t+1) k_{t+1}=n \sum_{T \in \mathcal{K}_{t}} D(T) .
$$

Hence, (12) is true, by expanding the right hand side of the above equation. For $2 \leq s<p$, let $f_{s}$ and $f_{s+1}$ be (12) with $t=s$ and $t=s+1$ respectively. Then (13) follows by considering $(p-s+1) f_{s}-$ $(s-1) \beta f_{s+1}$.

Now let $G^{\prime}=G \backslash V_{p-1}$. Notice that $G^{\prime}$ is $(1-2 \beta) n$-regular with $(1-\beta) n$ vertices. We observe that $G^{\prime}$ is a member of $\mathcal{G}\left(n^{\prime}, \beta^{\prime}\right)$, where $n^{\prime}=(1-\beta) n$ and $\beta^{\prime}=\beta /(1-\beta)$. Observe that $\left\lceil\beta^{\prime-1}\right\rceil-1=p-1$, so $1 / p \leq \beta^{\prime}<1 /(p-1)$. Recall that $k_{t}(G)=g_{t}(\beta) n^{t}$ for all $2 \leq$ $t \leq p$, so $k_{p+1}(G) g_{p}(\beta)=k_{p}(G) g_{p+1}(\beta) n$. Similarly, $k_{p}\left(G^{\prime}\right) g_{p-1}\left(\beta^{\prime}\right)=$ $k_{p-1}\left(G^{\prime}\right) g_{p}\left(\beta^{\prime}\right) n$. By considering $\mathcal{K}_{p}(G)$ and $\mathcal{K}_{p+1}(G)$, we obtain the following two equations :

$$
\begin{aligned}
k_{p+1}(G) & =\beta n k_{p}\left(G^{\prime}\right), \\
k_{p}(G) & =\beta n k_{p-1}\left(G^{\prime}\right)+k_{p}\left(G^{\prime}\right)=\beta n \frac{g_{p-1}\left(\beta^{\prime}\right) k_{p}\left(G^{\prime}\right)}{n^{\prime} g_{p}\left(\beta^{\prime}\right)}+k_{p}\left(G^{\prime}\right) \\
& =\left(1+\frac{\beta g_{p-1}\left(\beta^{\prime}\right)}{(1-\beta) g_{p}\left(\beta^{\prime}\right)}\right) k_{p}\left(G^{\prime}\right) .
\end{aligned}
$$

By substituting (15) and (16) into $k_{p}(G) n / k_{p+1}(G)=g_{p}(\beta) / g_{p+1}(\beta)$, we obtain (14). The proof is complete.

\section{$5 \quad K_{p+2}$-free graphs}

In this section, all graphs are assumed to be $K_{p+2}$-free. Lemma4.1implies that these graphs are also heavy-free. This means that $D_{+}(T)=$ 0 and $D(T) \leq(p-t+1) \beta$ for all $T \in \mathcal{K}_{t}$ and $t \leq p+1$. We prove the theorem below, which easily implies Theorem 2.2 as $g_{2}(\beta) n^{2}=$ $(1-\beta) n^{2} / 2 \leq k_{2}(G)$.

Theorem 5.1. Let $0<\beta<1$ and $p=\left\lceil\beta^{-1}\right\rceil-1$. Suppose $G$ is a $K_{p+2}$-free graph of order $n$ with minimum degree $(1-\beta) n$. Then

$$
\frac{k_{s}(G)}{g_{s}(\beta) n^{s}} \geq \frac{k_{t}(G)}{g_{t}(\beta) n^{t}}
$$

holds for $2 \leq t<s \leq p+1$. Moreover, the following three statements are equivalent:

(i) Equality holds for some $2 \leq t<s \leq p+1$.

(ii) Equality holds for all $2 \leq t<s \leq p+1$. 
(iii) The pair $(n, \beta)$ is feasible and $G$ is a member of $\mathcal{G}(n, \beta)$.

Proof. Fix $\beta$ and write $g_{t}$ to denote $g_{t}(\beta)$. Recall that $D_{+}(T)=0$ for cliques T. By Corollary 4.5 and Lemma 4.6, we have

$$
k_{t+1} \geq \frac{(1-t \beta)(p-t+1) \beta n k_{t}+(t-1)(t+2) k_{t+2} / n}{t-1+(p-2 t+2)(t+1) \beta}
$$

First, we are going to prove (17). It is sufficient to prove the case when $s=t+1$. We proceed by induction on $t$ from above. For $t=p$, $k_{p+2}=0$ and so (18) becomes

$$
(p-1-(p-2)(p+1) \beta) k_{p+1} \geq(1-p \beta) \beta n k_{p} .
$$

Since $g_{p+2}=0$, we have $k_{p+1} / g_{p+1} n^{p+1} \geq k_{p} / g_{p} n^{p}$ by (13). Hence, (17) is true for $t=p$. For $t<p$, (18) becomes

$$
\begin{aligned}
& (t-1+(t+1)(p-2 t+2) \beta) k_{t+1} \\
\geq & (1-t \beta)(p+1-t) \beta n k_{t}+(t-1)(t+2) k_{t+2} / n
\end{aligned}
$$

by the induction hypothesis

$$
\geq(1-t \beta)(p+1-t) \beta n k_{t}+(t-1)(t+2) g_{t+2} k_{t+1} / g_{t+1} .
$$

Thus, (17) follows from (13).

It is clear that (iii) implies both $(i)$ and $(i i)$ by Definition 1.1 and the feasibility of $(n, \beta)$. Suppose $(i)$ holds, so equality holds in (17) for $t=t_{0}$ and $s=s_{0}$ with $t_{0}<s_{0}$. We claim that equality must also hold for $t=p$ and $s=p+1$. Suppose the claim is false and equality holds for $t=t_{0}$ and $s=s_{0}$, where $s_{0}$ is maximal. Since equality holds for $t=t_{0}$, by (17), equality holds for $t=t_{0}, \ldots, s_{0}-1$ with $s=s_{0}$. We may assume that $t=s_{0}-1$ and $s_{0} \neq p+1$ and $k_{s_{0}+1} / g_{s_{0}+1} n>k_{s_{0}} / g_{s_{0}}$. However, this would imply a strictly inequality in (19) contradicting the fact that equality holds for $s=s_{0}$ and $t=s_{0}-1$. Thus, the proof of the claim is complete, that is, if $(i)$ holds then equality holds in (17) for $t=p$ and $s=p+1$.

Therefore, in order to prove that $(i)$ implies $(i i i)$, it is sufficient to show that if $k_{p+1} / g_{p+1} n^{p+1}=k_{p} / g_{p} n^{p}$, then $(n, \beta)$ is feasible and $G$ is a member of $\mathcal{G}(n, \beta)$. We proceed by induction on $p$. It is true for $p=2$ by Theorem 3 , so we may assume $p \geq 3$. Since equality holds in (17), we have equality in (18), Corollary 4.5 and Lemma 4.6. Since $D_{+}$is a zero function, equality in Corollary 4.5 implies equality in Corollary 4.3 and so $G$ is $(1-\beta) n$-regular as every vertex is a $(p+1)$-clique. In addition, for each $T \in \mathcal{K}_{p}$, either $D(T)=1-p \beta$ or $D(T)=\beta$ by equality in Lemma 4.6. Moreover, Corollary 4.3 implies 
that $\sum_{T \in \mathcal{K}_{p}(S)} D(T) \geq 2-(p+1) \beta$ for $S \in \mathcal{K}_{p+1}$. Thus, there exists $T \in \mathcal{K}_{p}(S)$ with $D(T)=\beta$. Pick $T \in \mathcal{K}_{p}$ with $D(T)=\beta$ and let $W=\bigcap\{N(v): v \in V(S)\}$, so $|W|=\beta$. Since $G$ is $K_{p+2}$-free, $W$ is a set of independent vertices. For each $w \in W, d(w)=(1-\beta) n$, so $N(w)=V(G) \backslash W$. Thus, the graph $G^{\prime}=G[V(G) \backslash W]$ is $\left(1-\beta^{\prime}\right) n^{\prime}-$ regular, where $n^{\prime}=(1-\beta) n, \beta^{\prime} n^{\prime}=(1-2 \beta) n$ and $\beta^{\prime}=\beta /(1-\beta)$. Note that $\left\lceil\beta^{\prime-1}\right\rceil-1=p-1$. Since $G$ is $K_{p+2}$-free, $G^{\prime}$ is $K_{p+1}$-free. Also, $k_{p+1}(G)=\beta n k_{p}\left(G^{\prime}\right)$ and

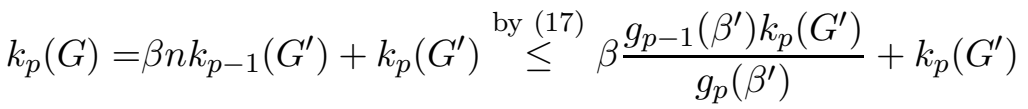

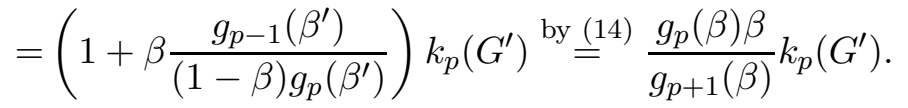

Hence,

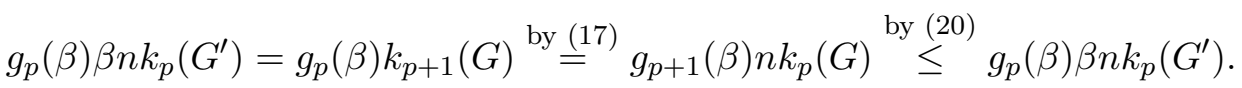

Therefore, we have $k_{p}\left(G^{\prime}\right) / g_{p}\left(\beta^{\prime}\right) n^{\prime p}=k_{p-1}\left(G^{\prime}\right) / g_{p-1}\left(\beta^{\prime}\right) n^{\prime p-1}$. By the induction hypothesis, $G^{\prime} \in \mathcal{G}\left(n^{\prime}, \beta^{\prime}\right)$, which implies $G \in \mathcal{G}(n, \beta)$. This completes the proof of the theorem.

\section{$6 k_{r}(n, \delta)$ for $2 n / 3<\delta \leq 3 n / 4$}

By Theorem 2.2, in order to prove Conjecture 1.2 it remains to handle the heavy cliques. However, even though both Corollary 4.5 and Lemma 4.6 are sharp by considering $G \in \mathcal{G}(n, \beta)$, they are not sufficient to prove Conjecture 1.2 even for the case when $2 n / 3<\delta \leq 3 n / 4$ by the observation below. Let $2 n / 3<\delta \leq 3 n / 4,1 / 4 \leq \beta<1 / 3$ and $p=3$. By Corollary 4.5 and Lemma 4.6, we have

$$
\begin{gathered}
(1+3 \beta) k_{3}+\sum_{T \in \mathcal{K}_{3}} D_{+}(T) \geq 2(1-2 \beta) \beta n k_{2}+\frac{4}{n} k_{4}+(1-2 \beta) n \sum_{e \in \mathcal{K}_{2}} D_{+}(e), \\
(2-4 \beta) k_{4}+2 \sum_{S \in \mathcal{K}_{4}} D_{+}(S) \geq(1-3 \beta) \beta n k_{3}+\frac{10}{n} k_{5}+(1-3 \beta) n \sum_{T \in \mathcal{K}_{3}} D_{+}(T),
\end{gathered}
$$

for $t=2$ and $t=3$ respectively. Since $D_{-}$is a zero function on 4-cliques,

$$
\sum_{S \in \mathcal{K}_{4}} D_{+}(S)=\sum_{S \in \mathcal{K}_{4}} D(S)=5 k_{5} / n
$$


Hence, the terms with $k_{5}$ and $\sum D_{+}(S)$ cancel in (22). Also, $(1-2 \beta)>$ 0 , so we may ignore the term with $\sum D_{+}(e)$ in (21). Recall that $g_{2}(\beta)=(1-\beta) / 2$ and $g_{3}(\beta)=(1-2 \beta)^{2} \beta$. After substitution of (22) into (21) replacing the $k_{4}$ term and rearrangement, we get

$$
k_{3}(G) \geq g_{3}(\beta) n^{3}-\frac{4 \beta-1}{1-\beta} \sum_{T \in \mathcal{K}_{3}} D_{+}(T) .
$$

However, $(4 \beta-1) \geq 0$ only if $\beta=1 / 4$. Hence, we are going to strengthen both (22) and (21). Recall that (21) is a consequence of Corollary 4.5 and Lemma 4.6 for $t=2$. Therefore, the following lemma, which is a strengthening of Corollary 4.5 for $t=2$, would lead to a strengthening of (21).

Lemma 6.1. Let $1 / 4 \leq \beta<1 / 3$. Suppose $G$ is a graph of order $n$ with minimum degree $(1-\beta) n$. Then, for $T \in \mathcal{K}_{3}$

$$
\widetilde{D}(T) \geq\left(1-\frac{2}{29-75 \beta}\right) \frac{4 \beta-1}{1-2 \beta} D_{+}(T)-(1-2 \beta) \sum_{e \in \mathcal{K}_{2}(T)} \frac{D_{+}(e)}{D_{+}(e)+\beta} .
$$

Moreover, if equality holds then $T$ is not heavy and $d(v)=(1-\beta) n$ for all $v \in T$.

Proof. Let $c$ be $(1-2 /(29-75 \beta))(4 \beta-1) /(1-2 \beta)$. Corollary 4.5 gives $\widetilde{D}(T) \geq 0$, so we may assume that $T$ is heavy. In addition, Corollary 4.3 implies that

$$
\widetilde{D}(T)+\sum_{e \in \mathcal{K}_{2}(T)} D_{+}(e) \geq D_{+}(T) .
$$

Since $c<1$, we may further assume that $T$ contains at least one heavy edge or else (23) holds as (24) becomes $\widetilde{D}(T) \geq D_{+}(T)>c D_{+}(T)$. Let $e_{0} \in \mathcal{K}_{2}(T)$ with $D_{+}\left(e_{0}\right)$ maximal. By substituting (24) into (23), it is sufficient to show that the function

$$
f=\left(1-\frac{1-2 \beta}{D_{+}\left(e_{0}\right)+2 \beta}\right) \widetilde{D}(T)-\left(c-\frac{1-2 \beta}{D_{+}\left(e_{0}\right)+2 \beta}\right) D_{+}(T)
$$

is non-negative.

First consider the case when $D_{+}(T) \leq 1-3 \beta$. Lemma 4.1 (iii) implies $D_{+}\left(e_{0}\right) \leq D_{+}(T) \leq 1-3 \beta$. Hence,

$$
\frac{1-2 \beta}{D_{+}\left(e_{0}\right)+2 \beta}-c \geq \frac{1-2 \beta}{1-\beta}-c>0 .
$$


Also, $1-2 \beta \leq 2 \beta<D_{+}\left(e_{0}\right)+2 \beta$. Therefore, $f>0$ by considering the coefficients of $\widetilde{D}(T)$ and $D(T)$. Hence, we may assume $D_{+}(T)>$ $1-3 \beta$. Since $T$ is heavy, $D_{-}(T)=\beta$. Therefore, by the definition of $\widetilde{D}$, we have

$$
\widetilde{D}(T)=\sum_{e \in \mathcal{K}_{2}(T)} D_{-}(e)-2(1-\beta) .
$$

We split into different cases separately depending on the number of heavy edges in $T$.

Suppose all edges are heavy. Thus, $\widetilde{D}(T)=2(4 \beta-1)$ by (25)), because $D_{-}(e)=2 \beta$ for all edges $e$ in $T$. Clearly $D_{+}(T)=D(T)-\beta \leq$ $1-\beta$. Hence, (23) is true as

$$
\widetilde{D}(T)=2(4 \beta-1) \geq(4 \beta-1)(1-\beta) /(1-2 \beta) \geq c D_{+}(T) .
$$

Thus, there exists an edge in $T$ that is not heavy and $D_{+}(T) \leq \beta$ by Lemma $4.1(v)$.

Suppose $T$ contains one or two heavy edges. We are going to show that in both cases

$$
\widetilde{D}(T) \geq 2\left(D_{+}(T)-(1-3 \beta)\right) .
$$

First assume that there is exactly one heavy edge in $T$. Let $e_{1}$ and $e_{2}$ be the two non-heavy edges in $T$. Note that $D_{-}\left(e_{i}\right)=D\left(e_{i}\right) \geq$ $D(T)=D_{+}(T)+\beta$ for $i=1,2$. Thus, (25) and Lemma 4.1 imply that $\widetilde{D}(T) \geq 2\left(D_{+}(T)-(1-3 \beta)\right)$. Assume that $T$ contains two heavy edges. Let $e_{1}$ be the non-heavy edge in $T$. Similarly, we have $D_{-}\left(e_{1}\right) \geq D_{+}(T)+\beta$. Recall that $D_{+}(T) \leq \beta$, so (25) and Lemma 4.1 imply

$$
\begin{aligned}
\widetilde{D}(T) & \geq\left(4 \beta+D_{+}(T)-(1-3 \beta)\right) \\
& =4 \beta-1+D_{+}(T)-(1-3 \beta) \geq 2\left(D_{+}(T)-(1-3 \beta)\right) .
\end{aligned}
$$

Since $\widetilde{D}(T) \geq 2\left(D_{+}(T)-(1-3 \beta)\right)$, in proving (23) , it is enough to show that

$$
\begin{aligned}
D\left(e_{0}\right) f= & \left(D_{+}\left(e_{0}\right)+2 \beta\right) f \\
\geq & 2\left(D_{+}\left(e_{0}\right)+4 \beta-1\right)\left(D_{+}(T)-(1-3 \beta)\right) \\
& -\left(\left(D_{+}\left(e_{0}\right)+2 \beta\right) c-(1-2 \beta)\right) D_{+}(T)
\end{aligned}
$$

is non-negative for $0<D_{+}\left(e_{0}\right) \leq D_{+}(T)$ and $1-3 \beta \leq D_{+}(T) \leq \beta$. Notice that for a fixed $D_{+}(T)$ it is enough to check the boundary points of $D_{+}\left(e_{0}\right)$. For $D_{+}\left(e_{0}\right)=0$, we have

$$
\begin{aligned}
D\left(e_{0}\right) f & \geq(2(3-c) \beta-1) D_{+}(T)-2(4 \beta-1)(1-3 \beta) \\
& \geq(4 \beta-1)\left(D_{+}(T)-(1-3 \beta)\right)>0 .
\end{aligned}
$$


For $D_{+}\left(e_{0}\right)=D_{+}(T)$, the right hand side of (26) becomes a quadratic function in $D_{+}(T)$. Moreover, both coefficients of $D_{+}(T)^{2}$ and $D_{+}(T)$ are positive. Thus, it enough to check for $D_{+}(T)=1-3 \beta$. For $D_{+}(T)=D_{+}\left(e_{0}\right)=1-3 \beta,(\underline{26})$ becomes

$$
D\left(e_{0}\right) f \geq(1-c-(2-c) \beta)(1-3 \beta)>0 .
$$

Hence, we have proved the inequality in Lemma 6.1.

It is easy to check that if equality holds in (23) then $D_{+}(T)=0$. Thus, for all edges $e$ in $T, D_{+}(e)=0$ by Lemma 4.1. Furthermore, equality holds in (24), so equality holds in Corollary 4.3 as $D_{+}(T)=$ $0=D_{+}(e)$. Hence, $d(v)=(1-\beta) n$ for $v \in S$. This completes the proof of the lemma.

Together with Lemma 4.6 with $t=2$, we obtain the strengthening of (21).

Corollary 6.2. Let $1 / 4 \leq \beta<1 / 3$. Suppose $G$ is a graph of order $n$ with minimum degree $(1-\beta) n$. Then

$$
(1+3 \beta) k_{3}+\frac{2}{1-2 \beta}\left(1-3 \beta+\frac{4 \beta-1}{29-75 \beta}\right) \sum_{T \in \mathcal{K}_{3}} D_{+}(T) \geq 2(1-2 \beta) \beta n k_{2}+4 \frac{k_{4}}{n}
$$

holds. Moreover, if equality holds, then $G$ is $(1-\beta) n$-regular and for each edge $e$, either we have $D(e)=1-2 \beta$ or $D(e)=2 \beta$.

Note that by mimicking the proof of Lemma 6.1, we could obtain a strengthening of Corollary 4.5 for $t=3$. It would lead to a strengthening of (22). However, it is still not sufficient to prove the Conjecture 1.2 when $\beta$ is close to $1 / 3$. Instead, we prove the following statement. The proof requires a detailed analysis of $\mathcal{K}_{5}$, so it is postponed to Section 7

Lemma 6.3. Let $1 / 4 \leq \beta<1 / 3$. Suppose $G$ is a graph order $n$ with minimum degree $(1-\beta) n$. Then

$$
(2-4 \beta) k_{4} \geq(1-3 \beta) \beta n k_{3}+\left(1-3 \beta+\frac{4 \beta-1}{29-75 \beta}\right) n \sum_{T \in \mathcal{K}_{3}} D_{+}(T) .
$$

Moreover, equality holds only if $(n, \beta)$ is feasible, and $G \in \mathcal{G}(n, \beta)$.

By using the two strengthened versions of (21) and (22), that is, Corollary 6.2 and Lemma 6.3, we prove the theorem below, which implies Theorem 2.3 . 
Theorem 6.4. Let $1 / 4 \leq \beta<1 / 3$. Let $s$ and $t$ be integers with $2 \leq$ $t<s \leq 4$. Suppose $G$ is a graph of order $n$ with minimum degree (1$\beta) n$. Then

$$
\frac{k_{s}(G)}{g_{s}(\beta) n^{s}} \geq \frac{k_{t}(G)}{g_{t}(\beta) n^{t}}
$$

Moreover, the following three statements are equivalent:

(i) Equality holds for some $2 \leq t<s \leq 4$.

(ii) Equality holds for all $2 \leq t<s \leq 4$.

(iii) The pair $(n, \beta)$ is feasible, and $G$ is a member of $\mathcal{G}(n, \beta)$.

Proof. Recall that $p=3$ as $1 / 4 \leq \beta<1 / 3$, so

$g_{2}(\beta)=(1-\beta) / 2, g_{3}(\beta)=(1-2 \beta)^{2} \beta$ and $g_{4}=(1-2 \beta)(1-3 \beta) \beta^{2} / 2$.

Note that in proving the inequality, it is sufficient to prove the case when $s=t+1$. Lemma 6.3 states that $(2-4 \beta) k_{4} \geq(1-3 \beta) \beta n k_{3}$. This implies $k_{4} / g_{4}(\beta) n^{4} \geq k_{3} / g_{3}(\beta) n^{3}$ by (13) with $t=3$. Hence, the theorem is true for $t=3$. For $t=2$, by substituting Corollary 6.2 into Lemma 6.3, we obtain

$$
\begin{array}{r}
(1+3 \beta) k_{3}+\frac{2}{1-2 \beta}\left(1-3 \beta+\frac{4 \beta-1}{29-75 \beta}\right) \sum_{T \in \mathcal{K}_{3}} D_{+}(T) \geq 2(1-2 \beta) \beta n k_{2} \\
+\frac{4}{(2-4 \beta) n}\left((1-3 \beta) \beta n k_{3}+\left(1-3 \beta+\frac{4 \beta-1}{29-75 \beta}\right) n \sum D_{+}(T)\right) .
\end{array}
$$

Observe that the $\sum D_{+}(T)$ terms on both sides cancel. Hence, after rearrangement, we have $(1-\beta) k_{3} \geq 2(1-2 \beta)^{2} \beta n k_{2}$. Thus, $k_{3} / g_{3}(\beta) n^{4} \geq k_{2} / g_{2}(\beta) n^{3}$ as required.

This is clear that $(i i i)$ implies $(i)$ and $(i i)$ by the construction of $\mathcal{G}(n, \beta)$ and the feasibility of $(n, \beta)$. Suppose $(i)$ holds, so equality holds for some $2 \leq t<s \leq 4$. It is easy to deduce that equality also holds for $s=4$ and $t=3$. By Lemma 6.3. $(n, \beta)$ is feasible, and $G \in \mathcal{G}(n, \beta)$.

\section{Proof of Lemma 6.3}

In this section, $T, S$ and $U$ always denote a 3-clique, 4-clique and 5 -clique respectively. Before presenting the proof, we recall some basic facts about $T, S$ and $U$. Observe that $D_{-}(S)=0$ for $S \in \mathcal{K}_{4}$, so $D_{+}(S)=D(S)$. Recall that $\widetilde{D}(S)=\sum_{T \in \mathcal{K}_{3}(S)} D_{-}(T)-(2-4 \beta)$. 
Let $T_{1}, \ldots, T_{4}$ be triangles in $S$ with $D\left(T_{i}\right) \leq D\left(T_{i+1}\right)$ for $1 \leq i \leq 3$. Since $D_{-}(T) \leq \beta$, we have

$$
\widetilde{D}(S)= \begin{cases}2(4 \beta-1) & \text { if } k_{3}^{+}(S)=4, \\ 4 \beta-1+\left(D\left(T_{1}\right)-(1-3 \beta)\right) & \text { if } k_{3}^{+}(S)=3, \\ D\left(T_{1}\right)+D\left(T_{2}\right)-2(1-3 \beta) & \text { if } k_{3}^{+}(S)=2,\end{cases}
$$

where $k_{3}^{+}(S)$ is the number of heavy triangles in $S$. Also recall that $D(T) \geq 1-3 \beta$ by Lemma $4.1(i)$. We will often make reference to these formulae throughout this section.

Proof of Lemma 6.3. Define the function $\eta: \mathcal{K}_{4} \rightarrow \mathbb{R}$ to be

$$
\eta(S)=\widetilde{D}(S)-\frac{4 \beta-1}{29-75 \beta} \sum_{T \in \mathcal{K}_{3}(S)} \frac{D_{+}(T)}{D_{+}(T)+\beta}
$$

for $S \in \mathcal{K}_{4}$. Recall that for a heavy triangle $T, D(T)=D_{+}(T)+\beta$. Thus, only heavy 3 -cliques in $S$ contribute to $\sum D_{+}(T) /\left(D_{+}(T)+\beta\right)$. We now claim that it is enough to show that $\sum_{S \in \mathcal{K}_{4}} \eta(S) \geq 0$. If $\sum_{S \in \mathcal{K}_{4}} \eta(S) \geq 0$, then Lemma 4.6 with $t=3$ implies that

$$
\begin{aligned}
0 \leq & \sum_{S \in \mathcal{K}_{4}} \eta(S)=\sum_{S \in \mathcal{K}_{4}} \widetilde{D}(S)-\frac{4 \beta-1}{29-75 \beta} n \sum_{T \in \mathcal{K}_{3}} D_{+}(T) \\
\leq & (2-4 \beta) k_{4}-(1-3 \beta) \beta n k_{3}-\left(1-3 \beta+\frac{4 \beta-1}{29-75 \beta}\right) n \sum_{T \in \mathcal{K}_{3}} D_{+}(T) \\
& +2 \sum_{S \in \mathcal{K}_{4}} D_{+}(S)-10 k_{5} / n,
\end{aligned}
$$

where the last inequality is due to Lemma4.6 with $t=3$. Observe that $\sum_{S \in \mathcal{K}_{4}} D_{+}(S)=\sum_{S \in \mathcal{K}_{4}} D(S)=5 k_{5} / n$, so the terms with $\sum D_{+}(S)$ and $k_{5} / n$ cancel. Rearranging the inequality, we obtain the inequality in Lemma 6.3.

Suppose $\sum_{S \in \mathcal{K}_{4}} \eta(S)<0$. Then, there exists a 4-clique $S$ with $\eta(S)<$ 0 . Such a 4-clique is called bad, otherwise it is called good. The sets of bad and good 4 -cliques are denoted by $\mathcal{K}_{4}^{\text {bad }}$ and $\mathcal{K}_{4}^{\text {good }}$ respectively. In the next claim, we identify the structure of a bad 4-clique.

Claim 7.1. Let $S$ be a bad 4-clique. Let

$$
\Delta=(1-3 \beta)(1+\epsilon) \text { and } \epsilon=(4 \beta-1) /\left(150 \beta^{2}-137 \beta+30\right) .
$$

Then, the following hold

(i) $S$ contains exactly one heavy edge and two heavy triangles,

(ii) $0<D(S)<\Delta$, 
(iii) $D(T)+D\left(T^{\prime}\right)<2 \Delta$, where $T$ and $T^{\prime}$ are the two non-heavy triangles in $S$.

Proof. Let $T_{1}, \ldots, T_{4}$ be triangles in $S$ with $D\left(T_{i}\right) \leq D\left(T_{i+1}\right)$ for $1 \leq$ $i \leq 3$. We may assume that $D_{+}\left(T_{4}\right)>0$, otherwise $S$ is good by Corollary 4.5 as $\eta(S)=\widetilde{D}(S) \geq 0$. Hence, $S$ is also heavy by Lemma 4.1 ( $i v)$. We separate cases by the number of heavy triangles in $S$.

First, suppose all triangles are heavy. Hence, $\widetilde{D}(S)=2(4 \beta-1)$ by (28). Clearly, $D_{+}\left(T_{i}\right) \leq 1-\beta$ for $1 \leq i \leq 4$, so

$$
\begin{aligned}
\eta(S) & \geq 2(4 \beta-1)-\frac{4 \beta-1}{29-75 \beta} \sum_{T \in \mathcal{K}_{3}(S)} \frac{D_{+}(T)}{D_{+}(T)+\beta} \\
& \geq 2(4 \beta-1)\left(1-\frac{2(1-\beta)}{29-75 \beta}\right)=\frac{2(4 \beta-1)(27-73 \beta)}{29-75 \beta} \geq 0 .
\end{aligned}
$$

This contradicts the assumption that $S$ is bad. Thus, not all triangles in $S$ are heavy, so $0<D(S) \leq \beta$ by Lemma $4.1(v)$. Also, $D_{+}(T) \leq$ $D_{+}(S)=D(S) \leq \beta$.

Suppose all but one triangles are heavy, so $\widetilde{D}(S) \geq 4 \beta-1$ by (28). Hence,

$$
\begin{aligned}
\eta(S) & \geq 4 \beta-1-\frac{4 \beta-1}{29-75 \beta} \sum_{T \in \mathcal{K}_{3}(S)} \frac{D_{+}(T)}{D_{+}(T)+\beta} \\
& \geq(4 \beta-1)\left(1-\frac{3}{29-75 \beta} \frac{D(S)}{D(S)+\beta}\right) \\
& \geq(4 \beta-1)\left(1-\frac{3}{2(29-75 \beta)}\right)=\frac{5(4 \beta-1)(11-30 \beta)}{2(29-75 \beta)} \geq 0,
\end{aligned}
$$

which is a contradiction.

Suppose there is only one heavy triangle, $T_{4}$, in $S$. Corollary 4.3 implies that $\widetilde{D}(S)+D_{+}\left(T_{4}\right) \geq 2 D_{+}(S)=2 D(S)$. Note that $D_{+}\left(T_{4}\right) \leq$ $D_{+}(S)=D(S)$, so $\widetilde{D}(S) \geq D(S)$. Thus,

$$
\begin{aligned}
\eta(S) & \geq D(S)-\frac{4 \beta-1}{29-75 \beta} \frac{D_{+}\left(T_{4}\right)}{D_{+}\left(T_{4}\right)+\beta} \geq D(S)-\frac{4 \beta-1}{29-75 \beta} \frac{D(S)}{D(S)+\beta} \\
& =\left(1-\frac{4 \beta-1}{(29-75 \beta)(D(S)+\beta)}\right) D(S) \geq\left(1-\frac{4 \beta-1}{(29-75 \beta) \beta}\right) D(S)>0 .
\end{aligned}
$$

Hence, $S$ has exactly two heavy triangles, namely $T_{3}$ and $T_{4}$. 
If $D(S) \geq \Delta$, then

$$
\begin{aligned}
\eta(S) & =D\left(T_{1}\right)+D\left(T_{2}\right)-2(1-3 \beta)-\frac{4 \beta-1}{29-75 \beta}\left(\frac{D_{+}\left(T_{3}\right)}{D_{+}\left(T_{3}\right)+\beta}+\frac{D_{+}\left(T_{4}\right)}{D_{+}\left(T_{4}\right)+\beta}\right) \\
& \geq 2(D(S)-(1-3 \beta))-\frac{2(4 \beta-1)}{29-75 \beta} \frac{D(S)}{D(S)+\beta} \\
& >2(1-3 \beta) \epsilon-\frac{2(4 \beta-1)}{29-75 \beta} \frac{\Delta}{\Delta+\beta} \\
& \geq 2(1-3 \beta) \epsilon-\frac{2(4 \beta-1) \Delta}{(29-75 \beta)(1-2 \beta)}=0 .
\end{aligned}
$$

Thus, $D(S)<\Delta$. If $D\left(T_{1}\right)+D\left(T_{2}\right) \geq 2 \Delta$, then $\widetilde{D}(S) \geq 2(\Delta-(1-$ $3 \beta))=2(1-3 \beta) \epsilon$ by (28). Moreover, since $D_{+}\left(T_{i}\right) \leq D(S)<\Delta$ for $i=3,4$,

$\eta(S)>2(1-3 \beta) \epsilon-\frac{2(4 \beta-1)}{29-75 \beta} \frac{\Delta}{\Delta+\beta} \geq 2(1-3 \beta) \epsilon-\frac{2(4 \beta-1) \Delta}{(29-75 \beta)(1-2 \beta)}=0$.

Thus, $(i i i)$ is true.

We have shown that $S$ contains exactly two heavy triangles. Therefore, to prove $(i)$, it is sufficient to prove that $S$ contains exactly one heavy edge. A triangle containing a heavy edge is heavy by Lemma 4.1 (iv). Since $S$ contains two heavy triangle, there is at most one heavy edge in $S$. It is enough to show that if $S$ does not contain any heavy edge and $D(S)<\Delta$, then $S$ is good, which is a contradiction. Assume that $S$ contains no heavy edge. Let $e_{i}=T_{i} \cap T_{4}$ be an edge of $T_{4}$ for $i=1,2,3$. We claim that $\widetilde{D}(S) \geq D_{+}\left(T_{4}\right)$. By Corollary 4.3 taking $S=T_{4}$ and $t=2$, we obtain

$$
\begin{aligned}
D\left(e_{1}\right)+D\left(e_{2}\right)+D\left(e_{3}\right) & \geq 2-3 \beta+D\left(T_{4}\right) \\
D\left(e_{1}\right)+D\left(e_{2}\right) & \geq 2-4 \beta+D_{+}\left(T_{4}\right) .
\end{aligned}
$$

as $D\left(e_{3}\right) \leq 2 \beta$ and $D_{-}\left(T_{4}\right)=\beta$. By Lemma $4.1(i i)$, we get

$$
D\left(T_{1}\right)+D\left(T_{2}\right) \geq D\left(e_{1}\right)+D\left(e_{2}\right)-2 \beta \geq 2(1-3 \beta)+D_{+}\left(T_{4}\right) .
$$

Hence, $\widetilde{D}(S) \geq D_{+}\left(T_{4}\right)$ by (28). Therefore,

$$
\begin{aligned}
\eta(S) & \geq D_{+}\left(T_{4}\right)-\frac{4 \beta-1}{29-75 \beta} \sum_{T \in \mathcal{K}_{4}(S)} \frac{D_{+}(T)}{D_{+}(T)+\beta} \\
& \geq\left(1-\frac{2(4 \beta-1)}{(29-75 \beta)\left(D_{+}\left(T_{4}\right)+\beta\right)}\right) D_{+}\left(T_{4}\right) \\
& \geq\left(1-\frac{2(4 \beta-1)}{(29-75 \beta) \beta}\right) D_{+}\left(T_{4}\right)>0
\end{aligned}
$$

and so $S$ is good, a contradiction. This completes the proof of the claim. 
Since a bad 4-clique $S$ must be heavy, that is, $D(S)>0$, it is contained in some 5-clique. A 5-clique is called bad if it contains at least one bad 4-clique. We denote $\mathcal{K}_{5}^{b a d}$ to be the set of bad 5-cliques. Define $\widetilde{\eta}(S)$ to be $\eta(S) / D(S)$ for $S \in \mathcal{K}_{4}$ with $D(S)>0$. Clearly,

$$
n \sum_{S \in \mathcal{K}_{4}} \eta(S)=\sum_{U \in \mathcal{K}_{5}} \sum_{S \in \mathcal{K}_{4}(U)} \widetilde{\eta}(S)+n \sum_{S \in \mathcal{K}_{4}: D(S)=0} \eta(S) .
$$

Recall that our aim is to show that $\sum_{S \in \mathcal{K}_{4}} \eta(S) \geq 0$. Since $D(S)=0$ implies that $S$ is good, we have $\eta(S) \geq 0$. Hence, it is enough to show that $\sum_{S \in \mathcal{K}_{4}(U)} \widetilde{\eta}(S) \geq 0$ for each bad 5-clique $U$.

Now, we give a lower bound on $\widetilde{\eta}(S)$ for bad 4-cliques $S$. By Claim 7.1,

$$
\eta(S) \geq-\frac{4 \beta-1}{29-75 \beta} \sum_{T \in \mathcal{K}_{3}(S)} \frac{D_{+}(T)}{D_{+}(T)+\beta} \geq-\frac{2(4 \beta-1)}{29-75 \beta} \frac{D(S)}{D(S)+\beta} .
$$

Hence,

$$
\widetilde{\eta}(S) \geq-\frac{2(4 \beta-1)}{(29-75 \beta)(D(S)+\beta)}>-\frac{2(4 \beta-1)}{(29-75 \beta) \beta} .
$$

Next, we are going to bound $D(S)$ above for $S \in \mathcal{K}_{4}(U) \backslash \mathcal{K}_{4}^{b a d}$ and $U \in \mathcal{K}_{5}^{b a d}$. Let $S^{b} \in \mathcal{K}_{4}^{b a d}(U)$. Observe that $S \cap S^{b}$ is a 3 -clique. Then, by Lemma 4.1 and Claim 7.1, we have

$$
D(S) \leq D\left(S \cap S^{b}\right)=D_{+}\left(S \cap S^{b}\right)+\beta \leq D\left(S^{b}\right)+\beta<\Delta+\beta .
$$

Recall that a bad 4-clique $S$ contains a heavy edge by Claim 7.1 and hence so does a bad 5-clique $U$. We split $\mathcal{K}_{5}^{\text {bad }}$ into subcases depending on the number of heavy edges in $U$. The next claim studies the relationship between the number of heavy edges and bad 4-cliques in a bad 5-clique $U$.

Claim 7.2. Let $U \in \mathcal{K}_{5}^{\text {bad }}$ with $h \geq 2$ heavy edges and $b$ bad 4-cliques. Then $b \leq 2 h /(h-1)=2+2 /(h-1)$. Moreover, if there exist two heavy edges sharing a common vertex, $b \leq 3$.

Proof. Define $H$ to be the graph induced by the heavy edges in $U$. Write $u_{S}$ for the vertex in $U$ not in $S \in \mathcal{K}_{4}(U)$. This defines a bijection between $V(U)$ and $\mathcal{K}_{4}(U)$. If $S$ is bad, $u_{S}$ is adjacent to all but one heavy edges by Claim 7.1, By summing the degrees of $H, 2 h=$ $\sum_{S \in \mathcal{K}_{4}(U)} d\left(u_{S}\right) \geq b(h-1)$. Thus, $b \leq 2 h /(h-1)$.

If there exist two heavy edges sharing a common vertex in $H$, then every bad 4-clique must miss one of the vertices of these two heavy edges. Hence, $b \leq 3$. 
Claim 7.3. Let $U \in \mathcal{K}_{5}^{b a d}$ with two heavy edges. Then $\sum_{S \in \mathcal{K}_{4}(U)} \widetilde{\eta}(S)>$ 0 .

Proof. Let $e$ and $e^{\prime}$ be two heavy edges in $U$, and let $b$ be the number of bad 4-cliques in $U$. We consider the cases whether $e$ and $e^{\prime}$ are vertex disjoint or not separately. First, assume that $e$ and $e^{\prime}$ are vertex disjoint. Notice that $\sum_{S \in \mathcal{K}_{4}^{\text {bad }}(U)} \widetilde{\eta}(S)>-b \gamma$ by (30), where $\gamma=2(4 \beta-1) /(29-75 \beta) \beta$ and $b \leq 4$ by Claim 7.2. Also, there is exactly one heavy 4-clique $S$ containing both $e$ and $e^{\prime}$. Therefore, it is sufficient to prove that $\eta(S) \geq b D(S) \gamma$. Since $S$ contains two disjoint heavy edges, all triangles in $S$ are heavy by Lemma 4.1 (iv). Thus, $\widetilde{D}(S)=2(4 \beta-1)$ by (28). Observe that $T=S \cap S^{\prime}$ is a triangle for $S^{\prime} \in \mathcal{K}_{4}(U) \backslash S$. Moreover, $D_{+}(T) \leq D_{+}\left(S^{\prime}\right)=D\left(S^{\prime}\right)$ by Lemma 4.1 (iii). Hence,

$$
\begin{aligned}
\eta(S) & \geq 2(4 \beta-1)-\frac{4 \beta-1}{29-75 \beta} \sum_{S^{\prime} \in \mathcal{K}_{4}(U) \backslash S} \frac{D\left(S^{\prime}\right)}{D\left(S^{\prime}\right)+\beta} \\
& >(4 \beta-1)\left(2-\frac{1}{29-75 \beta}\left(\frac{b \Delta}{\Delta+\beta}+\frac{(4-b)(\Delta+\beta)}{\Delta+2 \beta}\right)\right)
\end{aligned}
$$

by Claim 7.1 (ii) and (31). Therefore, $\eta(S)-b D(S) \gamma$ is at least

$$
\begin{aligned}
& (4 \beta-1)\left(2-\frac{1}{29-75 \beta}\left(\frac{b \Delta}{\Delta+\beta}+\frac{(4-b)(\Delta+\beta)}{\Delta+2 \beta}\right)\right)-b(\Delta+\beta) \gamma \\
\geq & (4 \beta-1)\left(2-\frac{4 \Delta}{(29-75 \beta)(\Delta+\beta)}\right)-4(\Delta+\beta) \gamma>0 .
\end{aligned}
$$

Thus, if $U$ contains two vertex disjoint heavy edges, $\sum_{S \in \mathcal{K}_{4}(U)} \widetilde{\eta}(S)>$ 0 . Similar argument also holds for the case when $e$ and $e^{\prime}$ share a common vertex.

Recall that a bad 5 -clique contains at least one heavy edge. Thus, we are left with the case $U \in \mathcal{K}_{5}^{\text {bad }}$ containing exactly one heavy edge.

Claim 7.4. Suppose $U \in \mathcal{K}_{5}^{\text {bad }}$ with exactly one heavy edge e. Then, $\sum_{S \in \mathcal{K}_{4}(U)} \widetilde{\eta}(S)>0$.

Proof. Let $u_{1}, \ldots, u_{5}$ be the vertices of $U$ with $u_{4} u_{5}$ is the heavy edge. Write $S_{i}$ and $\eta_{i}$ to be $U-u_{i}$ and $\eta\left(S_{i}\right)$ respectively for $1 \leq i \leq 5$. Similarly write $T_{i, j}$ to be $U-u_{i}-u_{j}$ for $1 \leq i<j \leq 5$. Recall that a bad 4-clique contains a heavy edge by Claim $7.1(i)$. Hence, $S_{i}$ is a bad 4-clique only if $i \leq 3$. Without loss of generality, $S_{1}, \ldots, S_{b}$ are the bad 4-cliques in $U$.

Since $S_{3}$ contains a heavy edge, it contains at least 2 heavy triangles by Lemma $4.1(i v)$. If $S_{3}$ contains either three or four heavy 
triangles, then $S_{3}$ is not bad by Claim $7.1(i)$. By a similar argument as in the proof of Claim 7.3, we can deduce that $\eta_{3} \geq 2 \gamma D\left(S_{3}\right)$, where as before $\gamma=2(4 \beta-1) /(29-75 \beta) \beta$. Therefore, $\sum_{S \in \mathcal{K}_{4}(U)} \widetilde{\eta}(S)>0$ as $b \leq 2$. Thus, we may assume that there are exactly two heavy triangles in $S_{i}$ for $1 \leq i \leq 3$. By Lemma $4.1(v), D\left(S_{i}\right)<\beta$ for $1 \leq i \leq 3$. For $1 \leq i \leq b$,

$$
D\left(T_{i, 4}\right)+D\left(T_{i, 5}\right)<2 \Delta=2(1-3 \beta)(1+\epsilon)
$$

by Claim 7.1 (iii). For $b<i \leq 3, \widetilde{D}\left(S_{i}\right)=D\left(T_{i, 4}\right)+D\left(T_{i, 5}\right)-2(1-3 \beta)$ by (28). Thus,

$$
\begin{aligned}
D\left(T_{i, 4}\right)+D\left(T_{i, 5}\right) & =\eta_{i}+2(1-3 \beta)+\frac{4 \beta-1}{29-75 \beta} \sum_{T \in \mathcal{K}_{3}\left(S_{i}\right)} \frac{D_{+}(T)}{D_{+}(T)+\beta} \\
& \leq \eta_{i}+2(1-3 \beta)+\frac{\gamma \beta D\left(S_{i}\right)}{D\left(S_{i}\right)+\beta} \\
& \leq \eta_{i}+2(1-3 \beta)+\gamma \beta / 2 .
\end{aligned}
$$

After applying Corollary 4.5 to $S_{4}$ and $S_{5}$ taking $t=3$, and adding the two inequalities together, we obtain

$$
\begin{aligned}
2(2-4 \beta) & \leq \sum_{1 \leq i \leq 3}\left(D_{-}\left(T_{i, 4}\right)+D_{-}\left(T_{i, 5}\right)\right)+2 D_{-}\left(T_{4,5}\right) \\
2(2-5 \beta) & \leq \sum_{1 \leq i \leq b}\left(D\left(T_{i, 4}\right)+D\left(T_{i, 5}\right)\right)+\sum_{b<i \leq 3}\left(D\left(T_{i, 4}\right)+D\left(T_{i, 5}\right)\right) \\
& <2 b(1-3 \beta)(1+\epsilon)+\sum_{b<i \leq 3} \eta_{i}+(3-b)(2(1-3 \beta)+\gamma \beta / 2) \\
2(4 \beta-1) & <2 b(1-3 \beta) \epsilon+\sum_{b<i \leq 3} \eta_{i}+(3-b) \gamma \beta / 2
\end{aligned}
$$

If $b=3$, the above inequality becomes $2(4 \beta-1)<6(1-3 \beta) \epsilon<2(4 \beta-$ 1 ), which is a contradiction. Thus, $b \leq 2$. Notice that $\eta_{i}>-D\left(S_{i}\right) \gamma>$ $-\gamma$ for $1 \leq i \leq b$. Hence, $\sum_{S \in \mathcal{K}_{4}^{\text {bad }}(U)} \widetilde{\eta}(S)>-b \gamma$. Also, recall that $D\left(S_{i}\right) \leq \beta$ for $1 \leq i \leq 3$. It is enough to show that $\sum_{b<i \leq 3} \eta_{i} \geq b \gamma \beta$. Suppose the contrary, so $\sum_{b<i \leq 3} \eta_{i}<b \gamma \beta$. Then, (32) becomes

$2(4 \beta-1)<2 b(1-3 \beta) \epsilon+(3+b) \gamma \beta / 2 \leq 4(1-3 \beta) \epsilon+5 \gamma \beta / 2<2(4 \beta-1)$,

which is a contradiction. The proof of the claim is complete.

Hence, by Claim 7.3 and Claim 7.4. (29) becomes $\sum_{S \in \mathcal{K}_{4}} \eta(S) \geq 0$, so the inequality in Lemma 6.3 holds. Now suppose equality holds in Lemma 6.3. Claim 7.3 and Claim 7.4 imply that no 5 clique is bad, so no 4-clique is bad. Furthermore, we must have $\eta(S)=0$ for 
all $S \in \mathcal{K}_{4}$. It can be checked that if the definition of a bad 4-clique includes heavy 4-cliques $S$ with $\eta(S)=0$, then all arguments still hold. Thus, we can deduce that $G$ is $K_{5}$-free. Hence, $G$ is also $K_{5}$-free. By Theorem 4 taking $s=4$ and $t=3$, we obtain that $(n, \beta)$ is feasible and $G \in \mathcal{G}(n, \beta)$.

\section{Proof of Theorem 2.4}

Here, we prove Theorem 2.4. Since the proof of theorem uses similar arguments in the proof of Theorem 5.1 and Lemma 6.1, we only give a sketch of the proof.

Sketch of Proof of Theorem 2.4. For $2 \leq t \leq p$ and $1 /(p+1) \leq \beta<$ $1 / p$, define

$$
\begin{aligned}
& A_{t}^{p}(\beta)=(t-1)((p+1) \beta-1) C_{t}^{p}(\beta), \text { and } \\
& B_{t}^{p}(\beta)=((p+1) \beta-1) C_{t}^{p}(\beta),
\end{aligned}
$$

where $C_{j}(\beta)$ satisfies the recurrence

$$
C_{t}(\beta)+1=(p-t+1) \beta C_{t-1}(\beta)
$$

with the initial condition $C_{p}(\beta)=0$ for $1 /(p+1) \leq \beta<1 / p$. Explicitly, $C_{p-j}^{p}(\beta)=\sum_{0 \leq i<j} i ! \beta^{i-j} / j$ ! for $0 \leq j \leq p-2$. These functions will be used as coefficients in corresponding statements of Lemma 6.1 for $2 \leq t<p$. Define the integer $r(\beta)$ to be the smallest integer at least 2 such that for $r \leq t \leq p, A_{t}^{p}(\beta)<1$ and $B_{t}^{p}(\beta)<(p-t) \beta$. Let

$$
\beta_{p}=\sup \left\{\beta_{0}: r(\beta)=2 \text { for all } 1 /(p+1) \leq \beta<\beta_{0}\right\}
$$

and $\epsilon_{p}=\beta_{p}-1 /(p+1)$. Observe that $A_{t}(\beta), B_{t}(\beta)$ and $C_{t}(\beta)$ are right continuous functions of $\beta$. Moreover, both $A_{t}(\beta)$ and $B_{t}(\beta)$ tend to zero as $\beta$ tends $1 /(p+1)$ from above, so $\beta_{p}>1 /(p+1)$ and $\epsilon_{p}>0$. By mimicking the poof of Lemma 6.1, we have

$$
\widetilde{D}(S) \geq A_{t+1}^{p}(\beta) D_{+}(S)-B_{t}^{p}(\beta) \sum_{T \in \mathcal{K}_{t}(S)} \frac{D_{+}(T)}{D(T)}
$$

for $S \in \mathcal{K}_{t+1}, 1 /(p+1) \leq \beta<\beta_{p}$ and $2 \leq t \leq p$. Then, following the arguments in the proof of Theorem 5.1, we can deduce that

$$
\frac{k_{s}(G)}{g_{s}(\beta) n^{s}} \geq \frac{k_{t}(G)}{g_{t}(\beta) n^{t}}+\frac{1-t \beta-B_{t}^{p}(\beta)}{(1-t \beta)(p-t+1) \beta g_{t}(\beta) n^{t}} \sum_{T \in \mathcal{K}_{t}} D_{+}(T)
$$

for $2 \leq t<s \leq p+1$ and $1 /(p+1)<\beta \leq \beta_{p}$. Since $1-t \beta-B_{t}^{p}(\beta) \geq 0$, the proof of theorem is completed.

Clearly, $\epsilon_{p}$ defined in the proof is not optimal. Generalising the proof of Lemma 6.3 would lead to an improvement on $\epsilon_{p}$. 


\section{Counting $(p+1)$-cliques}

In this section, we are going to prove the below theorem, which implies Theorem 2.5.

Theorem 9.1. Let $0<\beta<1$ and $p=\left\lceil\beta^{-1}\right\rceil-1$. Suppose $G$ is a graph of order $n$ with minimum degree $(1-\beta) n$. Then, for any integer $2 \leq t \leq p$,

$$
\frac{k_{p+1}(G)}{g_{p+1}(\beta) n^{p+1}} \geq \frac{k_{t}(G)}{g_{t}(\beta) n^{t}}
$$

Moreover, for $t=2$, equality holds if and only if $(n, \beta)$ is feasible, and $G$ is a member of $\mathcal{G}(n, \beta)$.

For positive integers $2 \leq t \leq s \leq p+1$, define the function $\phi_{t}^{s}$ : $\mathcal{K}_{s} \rightarrow \mathbb{R}$ such that

$$
\phi_{t}^{s}(S)= \begin{cases}D_{-}(S) & \text { if } t=s, \text { and } \\ \sum_{U \in K_{s-1}(S)} \phi_{t}^{s-1}(U) & \text { if } t<s\end{cases}
$$

for $S \in \mathcal{K}_{s}$. Observe that for $G_{0} \in \mathcal{G}(n, \beta)$ with $(n, \beta)$ feasible,

$$
\phi_{t}^{s}(S)= \begin{cases}(s-t) !(1-t \beta) & \text { if }\left|V(S) \cap V_{0}\right|=0,1 \\ (1-t \beta) s ! / t !+((p+1) \beta-1)(s-2) ! /(t-2) ! & \text { if }\left|V(S) \cap V_{0}\right|=2\end{cases}
$$

for $s$-cliques $S$ in $G_{0}$. Let $\Phi_{t}^{s}(S)=\min \left\{\phi_{t}^{s}(S), \varphi_{t}^{s}\right\}$ for $S \in \mathcal{K}_{s}$ and $2 \leq t \leq s \leq p+1$, where

$$
\varphi_{t}^{s}=(1-t \beta) s ! / t !+((p+1) \beta-1)(s-2) ! /(t-2) ! .
$$

to be the analogue of $D_{-}$for $\phi_{t}^{s}$. The next lemma gives a lower bound on $\Phi_{t}^{s}(S)$ for $S \in \mathcal{K}_{s}$.

Lemma 9.2. Let $0<\beta<1$ and $p=\left\lceil\beta^{-1}\right\rceil-1$. Let $G$ be a graph of order $n$ with minimum degree $(1-\beta) n$. Then,

$$
\Phi_{t}^{s}(S) \geq(1-t \beta) s ! / t !+\left(D_{-}(S)-(1-s \beta)\right)(s-2) ! /(t-2) !
$$

for $S \in \mathcal{K}_{s}$ and $2 \leq t<s \leq p+1$. In particular, for $s=p+1$ and $t=p$

$$
\sum_{S \in K_{p+1}} \Phi_{t}^{p+1}(S) \geq\left((1-t \beta) \frac{(p+1) !}{t !}-(1-(p+1) \beta) \frac{(p-1) !}{(t-2) !}\right) k_{p+1} .
$$


Proof. Fix $\beta$ and $t$ and we proceed by induction on $s$. The inequality holds for $s=t+1$ by Corollary 4.5. Suppose $s \geq t+2$ and that the lemma is true for $t, \ldots, s-1$. Hence

$\phi_{t}^{s}(S)=\sum_{T \in \mathcal{K}_{s-1}(S)} \phi_{t}^{s-1}(T) \geq \sum_{T \in \mathcal{K}_{s-1}(S)} \Phi_{t}^{s-1}(T)$

and by the induction hypothesis,

$$
\begin{aligned}
& \geq \sum_{T \in \mathcal{K}_{s-1}(S)}\left((1-t \beta) \frac{(s-1) !}{t !}+\left(D_{-}(T)-(1-(s-1) \beta)\right) \frac{(s-3) !}{(t-2) !}\right) \\
& =(1-t \beta) \frac{s !}{t !}+\frac{(s-3) !}{(t-2) !}\left(\sum_{T \in \mathcal{K}_{s-1}(S)} D_{-}(T)-s(1-(s-1) \beta)\right) \\
& \geq(1-t \beta) s ! / t !+\left(D_{-}(S)-(1-s \beta)\right)(s-2) ! /(t-2) !,
\end{aligned}
$$

where the last inequality comes from Corollary 4.5 with $t=s-1$. The right hand side is increasing in $D_{-}(S)$. In addition, the right hand side equals to $\varphi_{t}^{s}$ only if $D_{-}(S)=(p-s+1) \beta$. Thus, the proof of the lemma is complete.

Now, we bound $\sum_{S \in \mathcal{K}_{s}} \Phi_{t}^{s}(S)$ from above using Proposition 3.1 to obtain the next lemma. The proof is essentially a straightforward application of Proposition 3.1 with an algebraic check.

Lemma 9.3. Let $0<\beta<1$ and $p=\left\lceil\beta^{-1}\right\rceil-1$. Let $G$ be a graph of order $n$ with minimum degree $(1-\beta) n$. Then, for $2 \leq t \leq s \leq p+1$

$$
\begin{aligned}
\sum_{S \in \mathcal{K}_{s}} \Phi_{t}^{s}(S) \leq & \varphi_{t}^{s-1} s k_{s}+2((p+1) \beta-1) \sum_{i=t+1}^{s-1}\left(\frac{(i-3) !}{(t-2) !} k_{i} n^{s-i} \prod_{j=i}^{s-1}(1-j \beta)\right) \\
& +\left((t+1) k_{t+1}-(p-t+1) \beta k_{t} n\right) n^{s-t-1} \prod_{j=t}^{s-1}(1-j \beta) .
\end{aligned}
$$

Proof. Fix $\beta$ and $t$. We proceed by induction on $s$. Suppose $s=t+1$. Note that $\Phi_{t}^{t+1}(S) \leq \sum_{T \in \mathcal{K}_{t}(S)} D_{-}(T)$. By Proposition 3.1 taking $\mathcal{A}=\mathcal{K}_{t}, f=D_{-}, g=D, M=(p-t+1) \beta$ and $m=1-t \beta$,

$$
\begin{aligned}
& \sum_{S \in \mathcal{K}_{t+1}} \Phi_{t}^{t+1}(S) \leq \sum_{S \in \mathcal{K}_{t+1}} \sum_{T \in \mathcal{K}_{t}(S)} D_{-}(T)=n \sum_{T \in \mathcal{K}_{t}} D(T) D_{-}(T) \\
\leq & (p-t+1) \beta n \sum_{T \in \mathcal{K}_{t}} D(T)+(1-t \beta) n \sum_{T \in \mathcal{K}_{t}} D_{-}(T)-(1-t \beta)(p-t+1) \beta n k_{t} \\
\leq & (t+1)(1-(p-2 t+1) \beta) k_{t+1}-(1-t \beta)(p-t+1) \beta n k_{t} .
\end{aligned}
$$


Hence, the lemma is true for $s=t+1$. Now assume that $s \geq t+2$ and the lemma is true up to $s-1$. By Proposition 3.1 taking $\mathcal{A}=\mathcal{K}_{t}$, $f=\Phi_{t}^{s-1}, g=D, M=\varphi_{t}^{s-1}$ and $m=1-(s-1) \beta$, we have

$$
\begin{aligned}
& \sum_{S \in \mathcal{K}_{s}} \Phi_{t}^{s}(S)=n \sum_{T \in \mathcal{K}_{s-1}} D(T) \Phi_{t}^{s-1}(T) \\
\leq & \varphi_{t}^{s-1} \sum_{T \in \mathcal{K}_{s-1}} n D(T)+(1-(s-1) \beta) n \sum_{T \in \mathcal{K}_{s-1}} \Phi_{t}^{s-1}(T)-\varphi_{t}^{s-1}(1-(s-1) \beta) n k_{s-1} \\
= & \varphi_{t}^{s-1} s k_{s}+(1-(s-1) \beta) n \sum_{T \in \mathcal{K}_{s-1}} \Phi_{t}^{s-1}(T)-\varphi_{t}^{s-1}(1-(s-1) \beta) n k_{s-1} .
\end{aligned}
$$

Next, we apply induction hypothesis on $\sum \Phi_{t}^{s-1}(T)$. Note that

$$
(s-1) \varphi_{t}^{s-2}-\varphi_{t}^{s-1}=2((p+1) \beta-1)(s-4) ! /(t-2) ! .
$$

After collecting the terms, we obtain the desire inequality.

Now we are ready to prove Theorem 9.1. The proof is very similar to the proof of Theorem 5.1 .

Proof of Theorem 9.1. We fix $\beta$ and write $g_{t}$ to be $g_{t}(\beta)$. We proceed by induction on $t$ from above. The theorem is true for $t=p$ by Lemma 9.2 and Lemma 9.3 . Hence, we may assume $t<p$. By Lemma 9.3 ,

$$
\begin{aligned}
\sum \Phi_{t}^{p+1}(S) \leq & (p+1) \varphi_{t}^{p} k_{p+1}+2((p+1) \beta-1) \sum_{i=t+1}^{p}\left(\frac{(i-3) !}{(t-2) !} k_{i} n^{p+1-i} \prod_{j=i}^{p}(1-j \beta)\right) \\
& +\left((t+1) k_{t+1}-(p-t+1) \beta n k_{t}\right) n^{p-t} \prod_{j=t}^{p}(1-j \beta),
\end{aligned}
$$

and by the induction hypothesis

$$
\begin{aligned}
\leq & (p+1) \varphi_{t}^{p} k_{p+1}+2((p+1) \beta-1) \sum_{i=t+1}^{p}\left(\frac{k_{p+1} g_{i}}{g_{p+1}} \frac{(i-3) !}{(t-2) !} \prod_{j=i}^{p}(1-j \beta)\right) \\
& +\left((t+1) \frac{k_{p+1}}{g_{p+1}} g_{t+1}-(p-t+1) \beta n k_{t}\right) n^{p-t} \prod_{j=t}^{p}(1-j \beta) .
\end{aligned}
$$

Substitute the above inequality into (33) and rearranging to obtain the desire inequality.

Now suppose that equality holds, so equality holds in (33). Therefore, $D(S)=D_{-}(S)=0$ for all $S \in \mathcal{K}_{p+1}$. Thus, $G$ is $K_{p+2}$-free. By Theorem $5.1(n, \beta)$ is feasible, and $G \in \mathcal{G}(n, \beta)$. This completes the proof of the theorem. 


\section{Acknowledgements}

The author is greatly indebted to Andrew Thomason for his comments and his help in making the proof clearer.

\section{References}

[1] B. Andrásfai, P. Erdős, and V. T. Sós, On the connection between chromatic number, maximal clique and minimal degree of a graph, Discrete Math. 8 (1974), 205-218.

[2] B. Bollobás, On complete subgraphs of different orders, Math. Proc. Cambridge Philos. Soc. 79 (1976), 19-24.

[3] P. Erdős, On the number of complete subgraphs and circuits contained in graphs., Časopis Pěst. Mat. (1969), 290-296.

[4] D. Fisher, Lower bounds on the number of triangles in a graph, Journal of Graph Theory 13 (1989), 505-512.

[5] A. S. L. Lo, Triangles in regular graphs with density below one half, Combinatorics Probability and Computing 18 (2009), 435440.

[6] A. S. L. Lo, Cliques in graphs, Ph.D. thesis, University of Cambridge, 2010.

[7] L. Lovász and M. Simonovits, On complete subgraphs of a graph ii, Studies Pure Math. (1983), 459-496.

[8] V. Nikiforov, The number of cliques in graphs of given order and size, https://umdrive.memphis.edu/vnikifrv/public/pdfs/Ni07n.pdf, Preprint.

[9] A. Razborov, On the minimal density of triangles in graphs, Combinatorics, Probability and Computing (2008), 603-618.

[10] A. A. Razborov, Flag algebras, J. Symbolic Logic 72 (2007), $1239-1282$.

[11] P. Turán, Eine Extremalaufgabe aus der Graphentheorie, Mat. Fiz. Lapok 48 (1941), 436-452. 